\title{
Securing Visible Light Communication Systems by Beamforming in the Presence of Randomly Distributed Eavesdroppers
}

\author{
Sunghwan Cho, Student Member, IEEE, Gaojie Chen, Member, IEEE, and Justin P. Coon, Senior Member, IEEE
}

\begin{abstract}
This paper considers secrecy enhancement mechanisms in visible light communication (VLC) systems with spatially distributed passive eavesdroppers (EDs) under the assumption that there are multiple LED transmitters and one legitimate user equipment (UE). Based on certain amplitude constraints, we propose a beamforming scheme to improve secrecy performance. Contrary to the case where null-steering is made possible by using knowledge of the ED locations, the proposed beamforming when only statistical information about ED locations is available directs the transmission along a particular eigenmode related to the intensity of the ED process and the intended channel. Then, a LED selection scheme that is less complicated than beamforming is provided to reduce the secrecy outage probability (SOP). An approximate closed-form for the SOP is derived by using secrecy rate bounds. All the analysis is numerically verified by MonteCarlo simulations. The analysis shows that the beamformer yields superior performance to LED selection. However, LED selection is still a highly efficient alternative scheme due to the complexity associated with the use of multiple transmitters in the full beamforming approach. These performance trends and exact relations between system parameters can be used to develop a secure VLC system in the presence of randomly distributed EDs.
\end{abstract}

Index Terms-Physical layer security, visible light communication, beamforming, stochastic geometry, secrecy outage probability.

\section{INTRODUCTION}

D UE to the rapid proliferation of mobile communication devices and the associated difficulties in adequately allocating spectra to support new services, visible light communication (VLC) has become an increasingly interesting topic of research in academia and industry. The VLC medium does not interfere with RF systems, and VLC spectrum can be easily reused (spatially) since light can be confined to a certain indoor area. Moreover, VLC uses unregulated spectrum with a wide bandwidth (428 to $750 \mathrm{THz}$ ) and is capable of exploiting existing LED light infrastructure for communication [1], [2].

Compared to RF channels, VLC exploits line-of-sight (LoS) propagation and has relatively good signal confinement properties. However, the VLC channel is still of a broadcast nature. Therefore, securing VLC transmissions is an important issue, particularly for deployments in open places such as

S. Cho and J. P. Coon are with the Department of Engineering Science, University of Oxford, Oxford, OX1 3PJ, U.K. (e-mail: \{sunghwan.cho, justin.coon\}@eng.ox.ac.uk).

G. Chen is with the Department of Engineering, University of Leicester, Leicester, LE1 7RH, U.K. (e-mail: gaojie.chen@leicester.ac.uk).

This work was supported by EPSRC grant number EP/N002350/1 ("Spatially Embedded Networks"). public libraries, offices, and shopping malls. To cope with the security issue in RF systems, the focus on physical layer security (PLS), which is based on the information theoretic notion of employing coding to achieve secure communication, has accelerated since Wyner's seminal work [3]. Due to the broadcast nature of RF communications, both the legitimate receiver, or user equipment (UE), and eavesdroppers (EDs) may receive data from the source. However, the principle of PLS states that if the capacity of the intended data transmission channel is higher than that of the eavesdropping channel, the data can be transmitted at a rate close to the difference in their capacities, the so-called secrecy capacity, so that only the intended receiver can successfully decode the data [4].

It is difficult to obtain knowledge of passive ED locations. Yet, the analysis of secrecy capacity in spatial networks inherently depends upon this geometric properties. The mathematical theory of stochastic geometry is a powerful tool for dealing with spatial uncertainty [5], [6]. Using stochastic geometric methods, the impact of random ED locations on secrecy performance for RF communications has been investigated in recent years [7]-[10]. The location distribution of EDs can be modeled as a Poisson point process (PPP) or a binomial point process (BPP). In [7], the locations of multiple legitimate pairs and EDs were represented as independent two-dimensional PPPs, and the average secrecy throughput in such a wireless network was studied. Multiple-input multiple-output (MIMO) transmission with beamforming was considered later in [8], [9] to enhance secrecy performance. Transmit antenna selection and full-duplex schemes have also been used to enhance secrecy performance with randomly located EDs [10].

Motivated by the advantage of PLS, a recent topic of interest in the research community has been the investigation of PLS applied in VLC systems using various transmission methods, e.g., beamforming, jamming, etc. Recently, Mostafa et al. analyzed the achievable secrecy rate for single-input single-output (SISO) and multiple-input single-output (MISO) scenarios and proposed a variety of beamforming schemes such as zero-forcing (null-steering), artificial noise generation, friendly jamming, and robust beamforming [11]-[13]. Additionally, Zaid and Arfaoui proposed the truncated normal input distribution and the truncated generalized normal input distribution, respectively, to increase the secrecy rate under constraints on the input signal amplitude [14], [15]. It is important to note, however, that these contributions assumed a small number of EDs are present in the system and either the channel state information (CSI) or the locations of the EDs 
TABLE I. Notation and Symbols Used in the Paper

\begin{tabular}{|c|l|}
\hline Symbol & Definition/Explanation \\
\hline$L$ & the length of a room \\
$W$ & the width of a room \\
$Z$ & the height from the ceiling to the work plane \\
$N$ & number of transmitters \\
$\Phi_{E}$ & poisson point process of EDs \\
$\lambda_{E}$ & ED intensity function \\
$I_{D C}$ & fixed bias current \\
$R$ & photodetector's responsivity \\
$\alpha$ & modulation index \\
$\phi_{1 / 2}$ & half illuminance angle \\
$A_{P D}$ & physical area of a photodiode \\
$\phi$ & angle of irradiance \\
$\psi$ & angle of incidence \\
$K$ & refractive index of an optical concentrator \\
$\Psi_{C}$ & received field of view of a photodiode \\
$\mathbb{R}$ & set of real numbers \\
$\mathbb{R}_{+}$ & set of non-negative real numbers \\
$\mathbb{1}$ & all-ones column vector \\
$\mathbf{0}$ & all-zeros column vector \\
$\mathbb{E}[\cdot]$ & expectation operator \\
$\mathbb{P}(\cdot)$ & probability operator \\
{$[\cdot]^{T}$} & transpose operator \\
$\Gamma(x, y)$ & upper incomplete gamma function \\
\hline
\end{tabular}

are known. In practice, it might be impossible to obtain ED CSI or locations.

Inspired by the aforementioned contributions exploiting stochastic geometry in RF communications, our previous work [16] firstly developed an analogous approach to modeling ED locations in VLC systems. In this paper, we use this model to further analyze system performance and propose new MISO beamforming solutions. The contributions of this paper can be summarized comprehensively as follows:

- we propose a MISO beamforming solution that optimizes secrecy performance measures (e.g., the signal-to-noise ratio (SNR) and secrecy rate bounds) subject to a signal amplitude constraint for VLC systems when only information about the ED intensity measure is available at the transmitter;

- we demonstrate that the proposed beamforming method is well approximated by a simple LED selection scheme when the distance between the UE and one of the transmitting LEDs is small;

- we obtain closed-form bounds on the secrecy outage probability (SOP) when LED selection is adopted.

The rest of this paper is organized as follows ${ }^{1}$. Section II begins with the system model describing the modulation and beamforming schemes in VLC and providing various performance measures. In Section III, the beamformer maximizing secrecy performance is investigated. In Section IV, LED selection is proposed, and closed-form upper and lower bounds on the SOP are calculated. Section V gives numerical results that support our analysis. Section VI concludes the paper.

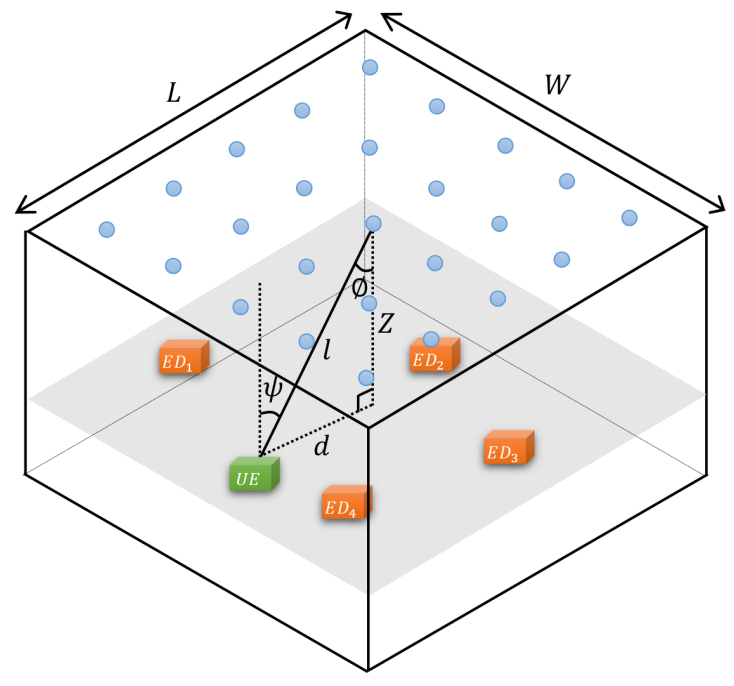

Fig. 1. Rectangular room configuration for VLC systems. $W$ and $L$ are the room's width and length, and $Z$ denotes the height from the ceiling to the work plane. Dots denote LED transmitters.

\section{System Model}

\section{A. Data Transmission}

We consider the downlink of a VLC system in a rectangular room $^{2}$ as shown in Fig. 1 , where $W, L$, and $Z$ denote the width, the length, and the height of the ceiling relative to the work plane, respectively. We assume that multiple UEs use the optical time-division multiple access (TDMA) [1] as the channel access scheme; thus only one fixed active UE can exist at a time. Also, multiple random passive EDs are assumed to be randomly distributed according to a PPP $\Phi_{E}$ with intensity $\lambda_{E}$ in the room. Note that there is no assumption that the PPP is homogeneous.

We assume all the receiver nodes are located on the same work plane, and $N$ transmitters are attached to the ceiling of the room. Each transmitter - i.e., an LED fixture consisting of multiple individual LEDs - is assumed to be capable of communicating independently of other transmitters [11]. We assume that EDs act independently of one another (i.e., there is no collusion).

A DC-biased pulse-amplitude modulation (PAM) VLC scheme is considered [11], [12]. The data signal $s_{i}(t) \in \mathbb{R}$ for the $i$ th transmitter in time slot $t$ is superimposed on a fixed bias current $I_{D C} \in \mathbb{R}_{+}$. The fixed bias $I_{D C}$ is used for the purpose of illumination. Thus, the $i$ th transmitter's modulated signal $x_{i}(t)$ of $s_{i}(t)$ is a zero-mean current signal that can be expressed by $x_{i}(t)=\alpha I_{D C} s_{i}(t)$, where $\alpha \in[0,1]$ is termed the modulation index. Note that $x_{i}(t)$ is the scaled version of $s_{i}(t)$. To maintain linear current-to-light conversion, the amplitude of $x_{i}(t)$ is constrained such that $\left|x_{i}(t)\right| \leq \alpha I_{D C}$. Thus, the dynamic range of the LED is $I_{D C} \pm \alpha I_{D C}$. Also, since $\mathbb{E}\left[x_{i}(t)\right]=0$, the modulated signal does not affect

\footnotetext{
${ }^{1}$ The notation and symbols used in the paper are listed in Table I.

${ }^{2}$ This may be an open space such as a shopping mall or a large office.
} 
illumination. Therefore, the MISO VLC channel model can be written as

$$
y(t)=\mathbf{h}^{T} \mathbf{x}(t)+n(t)
$$

where $\mathbf{h} \in\left[h_{1}, h_{2}, \ldots, h_{N}\right]^{T}$ is the channel gain vector, $\mathbf{x}(t)=$ $\left[x_{1}(t), x_{2}(t), \ldots, x_{N}(t)\right]^{T}$ is the transmitted signal vector, and $n(t)$ is the zero-mean additive white Gaussian noise (AWGN) at a receiver. Accordingly, the transmitted signal $\mathbf{x}(t)$ is subject to the amplitude constraint $|\mathbf{x}(t)| \leq \alpha I_{D C} \mathbb{1}$, where $\leq$ denotes the componentwise inequality. According to [17], the channel gain $h_{i} \in \mathbb{R}_{+}$in a VLC system corresponding to an LED with a generalized Lambertian emission pattern is given by

$h_{i}=\left\{\begin{array}{ccc}\eta \frac{(m+1) A_{P D}}{2 \pi l_{i}^{2}} \frac{\kappa^{2} \cos ^{m}\left(\phi_{i}\right)}{\sin ^{2}\left(\Psi_{c}\right)} \cos \left(\psi_{i}\right) R T & \text { for } & \left|\psi_{i}\right| \leq \Psi_{c} \\ 0 & \text { for } & \left|\psi_{i}\right|>\Psi_{c}\end{array}\right.$

where $\eta(W / A)$ is the current-to-light conversion efficiency and $m=-\ln (2) / \ln \left(\cos \left(\phi_{1 / 2}\right)\right)$ is the order of Lambertian emission with half illuminance at $\phi_{1 / 2}$, and $A_{P D}$ is the physical area of the photodiode (PD). As shown in Fig. 1, $l_{i}$ is the distance between the $i$ th transmitter and the receiver, and $d_{i}$ denotes the distance between the $i$ th transmitter and the receiver in the work plane. $\phi_{i}$ is the angle of irradiance, and $\psi_{i}$ is the angle of incidence. Also, $\kappa$ is the refractive index of the optical concentrator at the receiver, $\Psi_{c}$ denotes the received field of view of the PD, $R$ is the photodetector's responsivity, and $T(V / A)$ is the transimpedance amplifier gain. Note that this channel model considers only an LoS component and small scale fading does not exist in VLC systems since the detector area is much larger than the wavelength of visible light [18]. Moreover, by assuming that a receiver's PD faces up normal to the work plane, we can rewrite (2) in terms of $l_{i}$ as

$$
h_{i}=\eta \frac{(m+1) A_{P D}}{2 \pi l_{i}^{2}} \frac{\kappa^{2}}{\sin ^{2}\left(\Psi_{c}\right)}\left(\frac{Z}{l_{i}}\right)^{m}\left(\frac{Z}{l_{i}}\right) R T=K l_{i}^{-(m+3)}
$$

where $K=\left(\eta(m+1) A_{P D} Z^{m+1} \kappa^{2} R T\right) /\left(2 \pi \sin ^{2}\left(\Psi_{c}\right)\right)$.

As in [11], we define a beamforming vector $\mathbf{w}=$ $\left[w_{1}, w_{2}, \ldots, w_{N}\right]^{T}$, where $w_{i}$ for $i \in\{1,2, \ldots, N\}$ is a weight for the $i$ th transmitter and $\left|w_{i}\right| \leq 1$. Thus, the transmitted signal vector $\mathbf{x}(t)$ can be written as $\mathbf{x}(t)=\mathbf{w} \alpha I_{D C} s(t)$. Therefore, the received signal at the UE and eavesdropper $E_{e}$ for $e \in \Phi_{E}$ with the beamforming can be described as

$$
\begin{gathered}
y_{U}(t)=\alpha I_{D C} \mathbf{h}_{\mathbf{U}}^{\mathbf{T}} \mathbf{w} s(t)+n_{U}(t), \\
y_{E_{e}}(t)=\alpha I_{D C} \mathbf{h}_{\mathbf{E}_{\mathbf{e}}}^{\mathbf{T}} \mathbf{w} s(t)+n_{E_{e}}(t)
\end{gathered}
$$

respectively, where $\mathbf{h}_{\mathbf{U}}$ and $\mathbf{h}_{\mathbf{E}_{\mathbf{e}}} \in \mathbb{R}^{N}$ are the channel gain vectors from the transmitters to the $\mathrm{UE}$ and eavesdropper $E_{e}$, respectively, and $n_{U}$ and $n_{E_{e}}$ are zero-mean AWGN random variables at the UE and eavesdropper $E_{e}$, each with variance $\sigma^{2}$. For notational convenience, the time index $t$ is ignored for the remainder of the paper.

\section{B. Performance Measures}

For Gaussian VLC MISO channels with amplitude constraints, the peak SNR at the UE and the eavesdropper $E_{e}$ with the beamforming can be written as

$$
\begin{aligned}
\gamma_{U} & =\frac{\alpha^{2} I_{D C}^{2} \mathbf{w}^{\mathbf{T}} \mathbf{h}_{\mathbf{U}} \mathbf{h}_{\mathbf{U}}^{\mathbf{T}} \mathbf{w}}{\sigma^{2}}, \\
\gamma_{E_{e}} & =\frac{\alpha^{2} I_{D C}^{2} \mathbf{w}^{\mathbf{T}} \mathbf{h}_{\mathbf{E}_{\mathbf{e}}} \mathbf{h}_{\mathbf{E}_{\mathbf{e}}}^{\mathbf{T}} \mathbf{w}}{\sigma^{2}} .
\end{aligned}
$$

We use SNR to denote the peak, rather than average, SNR for the remainder of the paper. Note that this SNR is given only under the assumption of the proposed beamforming scheme. This is because the beamforming is not proven to be optimal for the Gaussian VLC MISO wiretap channel subject to amplitude constraints, unlike the Gaussian RF MISO wiretap channel considered in [19]. However, beamforming in VLC systems may be preferred as it permits a simple implementation [12]. Of course, other VLC MISO transmission schemes may also be considered, and it is hoped that this work will stimulate further investigations on this topic.

The capacity of the VLC channel is given by [20]

$$
C=\max _{p_{X}} \mathbb{I}(X ; Y)
$$

where $p_{X}$ is the input distribution and $\mathbb{I}(\cdot ; \cdot)$ denotes the mutual information. Note that the random variable $X$ has an amplitude constraint, i.e., $|X| \leq \alpha I_{D C}$. It is infeasible to calculate the closed-form solution for (6) due to this amplitude constraint [21]. Thus, the upper and lower bounds on the transmission rate under the beamfoming scheme proposed in (4) are used for our analysis, which are given in [20, Theorem 5] as

$$
\begin{aligned}
& C^{\text {upper }}=\frac{1}{2} \log (1+\gamma), \\
& C^{\text {lower }}=\frac{1}{2} \log \left(1+\frac{2 \gamma}{\pi e}\right)
\end{aligned}
$$

where $\gamma$ is the received SNR. Note that these bounds on the rate are based on the SISO channel [20], but we translate these bounds into the MISO channel by setting the input of the SISO channel as $\alpha I_{D C} \mathbf{h}^{\mathbf{T}} \mathbf{w} s(t)$ using the proposed beamforming scheme similarly in [22].

In addition to that, we define the SOP as the probability that the secrecy capacity $C_{s}$ is lower than a threshold secrecy rate $C_{\text {th }}$, i.e.,

$$
P_{\mathrm{SO}}=\mathbb{P}\left(C_{s} \leq C_{\mathrm{th}}\right) .
$$

However, since the closed-form of the secrecy capacity with the input amplitude constraint is also not readily available, we employ the lower and upper bounds on the secrecy rate as defined in [11, Theorem 1], which are given by

$$
\begin{aligned}
& C_{s}^{\text {lower }}=\max \left\{\frac{1}{2} \log \left(\frac{6 \gamma_{U}+3 \pi e}{\pi e \gamma_{E}^{*}+3 \pi e}\right), 0\right\}, \\
& C_{s}^{\text {upper }}=\max \left\{\frac{1}{2} \log \left(\frac{\gamma_{U}+1}{\gamma_{E}^{*}+1}\right), 0\right\}
\end{aligned}
$$

where $\gamma_{E}^{*}=\max _{e \in \Phi_{E}} \gamma_{E_{e}}$ (i.e., the worst case ED with the highest $\mathrm{SNR})$ and $e$ in (9a) is the base of the natural logarithm. Note that the upper bound also can be possible only under the proposed beamforming scheme. Applying these bounds yields 
the following upper and lower bounds on the SOP:

$$
\begin{aligned}
& P_{\text {SO }}^{\text {upper }}=\mathbb{P}\left(C_{s}^{\text {lower }} \leq C_{\mathrm{th}}\right), \\
& P_{\text {SO }}^{\text {lower }}=\mathbb{P}\left(C_{s}^{\text {upper }} \leq C_{\mathrm{th}}\right) .
\end{aligned}
$$

\section{BEAMFORMING}

In this section, we propose beamformer designs based on the formulation of several optimisation problems that aim to improve secrecy performance when only information about the intensity of the ED PPP is known. Crucially, we demonstrate that the proposed beamforming solutions apply to both homogenous and inhomogeneous ED processes.

\section{A. Beamforming Based on SNR}

Without knowledge of ED locations, a natural objective is to minimize the average SNR of EDs $\bar{\gamma}_{E}$ subject to a constraint on the minimum required UE SNR $\gamma_{U}$, same as in RF communications [23]-[25]. A related, alternative objective may be to maximize $\gamma_{U}$ subject to a constraint on $\bar{\gamma}_{E}$. In this subsection, both of these cases will be investigated.

1) Minimizing Average Eavesdropper SNR: The SNR of the UE (5a) can be written as

$$
\gamma_{U}=\varphi \mathbf{w}^{\mathbf{T}} \mathbf{A w}
$$

where $\varphi=\alpha^{2} I_{D C}^{2} / \sigma^{2}$ and $\mathbf{A}=\mathbf{h}_{\mathbf{U}} \mathbf{h}_{\mathbf{U}}^{\mathbf{T}}$. Note that the rank of A is one. Also, from (5b), the average SNR of an ED can be written as

$$
\bar{\gamma}_{E}=\mathbb{E}\left[\varphi \mathbf{w}^{\mathbf{T}} \mathbf{h}_{\mathbf{E}_{\mathrm{e}}} \mathbf{h}_{\mathbf{E}_{\mathrm{e}}}^{\mathbf{T}} \mathbf{w}\right]=\varphi \mathbf{w}^{\mathbf{T}} \mathbb{E}\left[\mathbf{h}_{\mathbf{E}_{\mathrm{e}}} \mathbf{h}_{\mathbf{E}_{\mathbf{e}}}^{\mathbf{T}}\right] \mathbf{w}=\varphi \mathbf{w}^{\mathbf{T}} \overline{\mathbf{B}} \mathbf{w}
$$

where $\overline{\mathbf{B}}=\mathbb{E}\left[\mathbf{h}_{\mathbf{E}_{\mathbf{e}}} \mathbf{h}_{\mathbf{E}_{\mathbf{e}}}^{\mathbf{T}}\right]$. The element in the $i$ th row and $j$ th column of $\overline{\mathbf{B}}$ is given by

$$
\bar{B}_{i, j}=\frac{1}{N_{E}} \int_{\frac{-L}{2}}^{\frac{L}{2}} \int_{\frac{-W}{2}}^{\frac{W}{2}} \frac{\lambda_{E}(x, y) K^{2}}{l_{i}^{m+3}(x, y) l_{j}^{m+3}(x, y)} \mathrm{d} x \mathrm{~d} y
$$

where $\lambda_{E}(x, y)$ is the intensity of EDs at the point $(x, y)$ and $l_{i}(x, y)$ for $i \in\{1,2, \cdots, N\}$ is the distance between the $i$ th transmitter and the point $(x, y)$. Note that $\lambda_{E}(x, y)$ is a constant when the ED point process is homogeneous. Also, $N_{E}$ denotes the average number of EDs, which is given by

$$
N_{E}=\int_{\frac{-L}{2}}^{\frac{L}{2}} \int_{\frac{-W}{2}}^{\frac{W}{2}} \lambda_{E}(x, y) \mathrm{d} x \mathrm{~d} y .
$$

From the formulation given above, it is clear that the beamforming vector $\mathbf{w}^{*}$ is given by

$$
\begin{aligned}
& \mathbf{w}^{*}=\underset{\mathbf{w}}{\arg \min _{\mathbf{w}} \varphi \mathbf{w}^{\mathbf{T}} \overline{\mathbf{B}} \mathbf{w}} \\
& \text { s.t. }\left\{\begin{array}{l}
\varphi \mathbf{w}^{\mathbf{T}} \mathbf{A w} \geq \rho_{U} \\
|\mathbf{w}| \leq \mathbb{1}
\end{array}\right.
\end{aligned}
$$

where $\rho_{U}$ denotes the required SNR of the UE. Note that the beamformer $\mathbf{w}^{*}$ is $\mathbf{0} \in \mathbb{R}^{N}$ without the first constraint. Then, we form the Lagrangian [26] as follows

$$
\mathcal{L}=\varphi \mathbf{w}^{\mathbf{T}} \overline{\mathbf{B}} \mathbf{w}-\lambda\left(\varphi \mathbf{w}^{\mathbf{T}} \mathbf{A w}-\rho_{U}\right)-\mu_{-}^{\mathbf{T}}(\mathbf{w}+\mathbb{1})+\mu_{+}^{\mathbf{T}}(\mathbf{w}-\mathbb{1})
$$

where $\lambda \in \mathbb{R}$ and $\mu_{-}, \mu_{+} \in \mathbb{R}^{N}$ are the Lagrange multipliers. To let $\mathcal{L}$ have the non-trivial minimum value with respect to w and analytically calculate the solution of (15), the condition has to be satisfied as

$$
\mu_{-}=\mu_{+}=\mathbf{0}
$$

According to the local sensitivity analysis in [26], zero Lagrange multipliers $\mu_{-}$and $\mu_{+}$imply that the second inequality constraint is slack, i.e., $\left|w_{i}\right| \neq 1$ for $i \in\{1,2, \ldots, N\}$.

If (17) can be satisfied ${ }^{3}$, computing the partial derivative of $\mathcal{L}$ with respect to $\mathbf{w}$ and setting the result equal to zero leads to

$$
(\overline{\mathbf{B}}-\lambda \mathbf{A}) \mathbf{w}=\mathbf{0} .
$$

The beamforming vector must satisfy (18). If $\lambda=0$ (i.e., the UE SNR constraint is inactive), w should belong to the null space of $\overline{\mathbf{B}}$. Referring to (13), it should be clear that the rank of $\overline{\mathbf{B}}$ depends on the intensity of the ED process. Indeed, it is possible that the intensity is such that $\overline{\mathbf{B}}$ is reduced rank. In this case, the vectors of the null space of $\overline{\mathbf{B}}$ yields $\overline{\mathbf{B}} \mathbf{w}=\mathbf{0}$, thus all the vectors of the null space of $\overline{\mathbf{B}}$ satisfying (15b) can be the solution of (15). On the other hand, if $\overline{\mathbf{B}}$ is full rank ${ }^{4}$, we have

$$
\overline{\mathbf{B}}^{-1} \mathbf{A} \mathbf{w}=\eta \mathbf{w}
$$

where $\eta=1 / \lambda$. This implies that $\eta$ is the eigenvalue of $\overline{\mathbf{B}}^{-1} \mathbf{A}$ and $\mathbf{w}$ is the corresponding eigenvector. Hence, the solution satisfies

$$
\bar{\gamma}_{E}=\varphi \mathbf{w}^{\mathbf{T}} \overline{\mathbf{B}} \mathbf{w}=\varphi \frac{1}{\eta} \mathbf{w}^{\mathbf{T}} \mathbf{A} \mathbf{w} \geq \frac{1}{\eta_{\max }} \rho_{U} .
$$

From this, we deduce that the minimum $\bar{\gamma}_{E}$ is inversely proportional to the maximum eigenvalue $\eta_{\max }$. Here, since $\mathbf{A}$ is rank one, we have that $\overline{\mathbf{B}}^{-\mathbf{1}} \mathbf{A}$ is rank one since

$$
0<\operatorname{rank}\left(\overline{\mathbf{B}}^{-\mathbf{1}} \mathbf{A}\right) \leq \min \{\operatorname{rank}(\overline{\mathbf{B}}), \operatorname{rank}(\mathbf{A})\}=1 .
$$

Hence, there exists a single non-zero eigenvalue $\eta_{\max }$, and the beamformer $\mathbf{w}^{*}$ is obtained by scaling the corresponding eigenvector such that $\varphi \mathbf{w}^{* \mathbf{T}} \mathbf{A} \mathbf{w}^{*}=\rho_{U}$ and $\left|\mathbf{w}^{*}\right|<\mathbb{1}$. When feasible, the fact that only one non-zero eigenvalue exists implies the solution is unique. Hence, it is clear that this approach gives a simple method of calculating the beamforming vector.

On the other hand, if $\rho_{U}$ is high so that (17) cannot be satisfied, the solution $\mathbf{w}^{*}$ can be found numerically with the convex-concave procedure (CCP) [27], which is a powerful heuristic method to find local solutions. Given a initial feasible point $\mathbf{w}_{0}$, we transform the first constraint in (15b) into the affine function (i.e., convex) by applying the first-order Taylor series approximation and solve the convex optimization problem. Then, we set the solution to $\mathbf{w}_{1}$ and repeat the same procedure until the improvement in the objective value is less than some threshold $\epsilon$. The detailed iterative algorithm is given in Algorithm 1.

\footnotetext{
${ }^{3}$ If $\rho_{U}$ can be achieved by only the transmission of the nearest LED to the UE, the beamformers of other transmitters must not be \pm 1 . Moreover, without loss of generality, we can assume that the beamforming element of the nearest LED can be very close to \pm 1 , but not equal to \pm 1 . Then, (17) can be satisfied.

${ }^{4}$ This condition can be confirmed when the ED PPP is homogeneous, for example.
} 


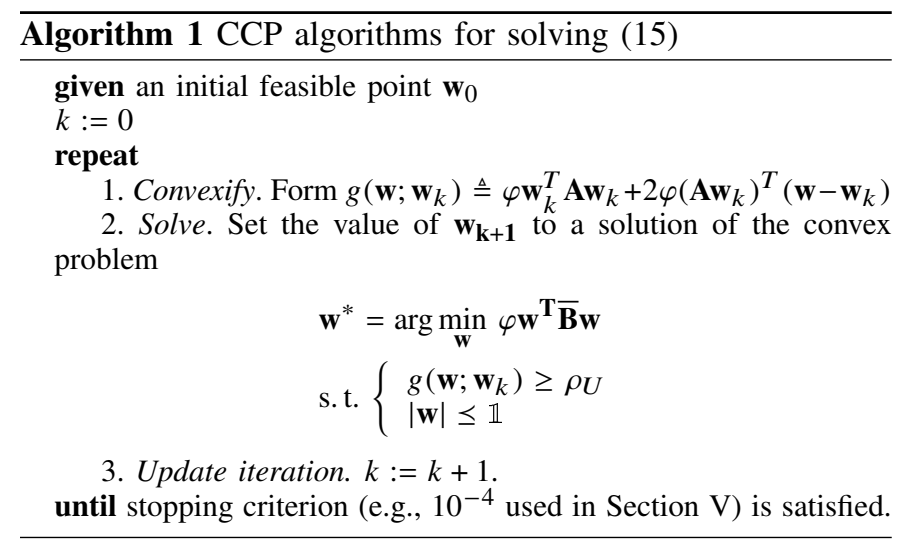

2) Maximizing User SNR: We now investigate the problem of maximizing the SNR of the UE $\gamma_{U}$ while constraining the average SNR of the EDs $\bar{\gamma}_{E}$. Similar to the previous subsection, we can formulate the optimization problem as

$$
\begin{aligned}
& \mathbf{w}^{*}=\arg \max _{\mathbf{w}} \varphi \mathbf{w}^{\mathbf{T}} \mathbf{A} \mathbf{w} \\
& \text { s.t. }\left\{\begin{array}{l}
\varphi \mathbf{w}^{\mathbf{T}} \overline{\mathbf{B}} \mathbf{w} \leq \bar{\rho}_{E} \\
|\mathbf{w}| \leq \mathbb{1}
\end{array}\right.
\end{aligned}
$$

where $\bar{\rho}_{E}$ is the target constraint on the average SNR of EDs.

Following the same approach as above leads to an analogous result:

$$
(\mathbf{A}-\lambda \overline{\mathbf{B}}) \mathbf{w}=\mathbf{0} .
$$

When $\overline{\mathbf{B}}$ is singular, unlike the previous approach, we cannot guarantee that the vectors of the null space of $\overline{\mathbf{B}}$ satisfying the constraint (22b) maximize the objective function (22a). Thus, in this case, this problem should be solved by a numerical method as proposed in Algorithm 1. If $\overline{\mathbf{B}}$ is nonsingular, we have

$$
\overline{\mathbf{B}}^{-\mathbf{1}} \mathbf{A} \mathbf{w}=\lambda \mathbf{w} .
$$

Hence, $\lambda$ and $\mathbf{w}$ are an eigenvalue-eigenvector pair for $\overline{\mathbf{B}}^{-\mathbf{1}} \mathbf{A}$. The solution satisfies

$$
\gamma_{U}=\varphi \mathbf{w}^{\mathbf{T}} \mathbf{A w}=\varphi \lambda \mathbf{w}^{\mathbf{T}} \overline{\mathbf{B}} \mathbf{w} \leq \lambda_{\max } \bar{\rho}_{E}
$$

where $\lambda_{\max }$ is the maximum (indeed, the only nonzero) eigenvalue, and the beamformer $\mathbf{w}^{*}$ is the associated eigenvector after being scaled such that $\varphi \mathbf{w}^{* \mathbf{T}} \overline{\mathbf{B}} \mathbf{w}^{*}=\bar{\rho}_{E}$ and $|\mathbf{w}| \prec \mathbb{1}$ (if feasible).

\section{B. Beamforming Based on Rate}

In this subsection, we will investigate beamforming based on the achievable transmission rate of the UE and EDs under the proposed beamforming scheme.

1) Minimizing Eavesdropper Average Rate: Since the closed-form expression for the capacity of a VLC channel is not available, we will use the upper and lower bounds given in (7). Thus, the beamformer $\mathbf{w}^{*}$ minimizing the average ED rate upper bound under the proposed beamforming is given by

$$
\mathbf{w}^{*}=\arg \min _{\mathbf{w}} \mathbb{E}\left[\frac{1}{2} \log \left(1+\gamma_{E_{e}}\right)\right]
$$

$$
\text { s.t. }\left\{\begin{array}{l}
\frac{1}{2} \log \left(1+\frac{2 \gamma_{U}}{\pi e}\right) \geq \xi_{U} \\
|\mathbf{w}| \leq \mathbb{1}
\end{array}\right.
$$

where $\xi_{U}$ is the required rate lower bound for the UE. However, since the objective function (26a) does not lend itself to tractable analysis and a practically implementable solution, we revise the objective function using Jensen's inequality, i.e., it becomes $(1 / 2) \log \left(1+\bar{\gamma}_{E}\right)$, which allows us to obtain the alternative beamformer $\mathbf{w}^{*}$. In addition, since $\log (\cdot)$ is a monotonic increasing function, (26) can be reformulated to

$$
\begin{aligned}
& \mathbf{w}^{*}=\arg \min _{\mathbf{w}} \varphi \mathbf{w}^{\mathbf{T}} \overline{\mathbf{B}} \mathbf{w} \\
& \text { s.t. }\left\{\begin{array}{l}
\varphi \mathbf{w}^{\mathbf{T}} \mathbf{A w} \geq M_{1} \\
|\mathbf{w}| \leq \mathbb{1}
\end{array}\right.
\end{aligned}
$$

where $M_{1}=\left(2^{2 \xi_{U}}-1\right) \pi e / 2$. Note that (27) has an identical form to (15). When $\overline{\mathbf{B}}$ is invertible, It follows that the altenative beamformer $\mathbf{w}^{*}$ is the eigenvector corresponding to the maximum eigenvalue of $\overline{\mathbf{B}}^{-\mathbf{1}} \mathbf{A}$ after being scaled such that $\varphi \mathbf{w}^{* \mathbf{T}} \mathbf{A} \mathbf{w}^{*}=M_{1}$ and $|\mathbf{w}|<\mathbb{1}$. If $\overline{\mathbf{B}}$ is reduced rank, the vectors of the null space of $\overline{\mathbf{B}}$ satisfying (27b) can be the solution of (27).

2) Maximizing User Rate: The optimization problem for maximizing the lower bound of the UE's rate subject to an ED rate constraint can be formulated as

$$
\begin{aligned}
& \mathbf{w}^{*}=\arg \max _{\mathbf{w}} \frac{1}{2} \log \left(1+\frac{2 \gamma_{U}}{\pi e}\right) \\
& \text { s.t. }\left\{\begin{array}{l}
\frac{1}{2} \mathbb{E}\left[\log \left(1+\gamma_{E_{e}}\right)\right] \leq \bar{\xi}_{E} \\
|\mathbf{w}| \leq \mathbb{1}
\end{array}\right.
\end{aligned}
$$

where $\bar{\xi}_{E}$ is the target constraint for the average rate upper bound of EDs. Again, by applying Jensen's inequality to the constraint, we arrive at the alternative formulation

$$
\begin{aligned}
& \mathbf{w}^{*}=\arg \max _{\mathbf{w}} \varphi \mathbf{w}^{\mathbf{T}} \mathbf{A} \mathbf{w} \\
& \text { s.t. }\left\{\begin{array}{l}
\varphi \mathbf{w}^{\mathbf{T}} \overline{\mathbf{B}} \mathbf{w} \leq M_{2} \\
|\mathbf{w}| \leq \mathbb{1}
\end{array}\right.
\end{aligned}
$$

where $M_{2}=2^{2 \bar{\xi}_{E}}-1$. Hence, if $\overline{\mathbf{B}}$ is invertible, we deduce that the alternative beamforming vector is the eigenvector corresponding to the maximum eigenvalue of $\overline{\mathbf{B}}^{-\mathbf{1}} \mathbf{A}$ scaled appropriately as similar as the problem (22). If $\overline{\mathbf{B}}$ is not invertible, the solution should be numerically found via the CCP algorithm proposed in Algorithm 1.

\section{Comparing MISO Beamforming to LED Selection}

In the previous subsection, we showed that the beamformer for SNR and rate objectives is universally related to the maximum eigenmode of $\overline{\mathbf{B}}^{-\mathbf{1}} \mathbf{A}$. The proposed beamforming vector cannot be a null-steering solution as was the case in [12] unless a (perhaps pathological) condition occurs that makes $\overline{\mathbf{B}}$ singular.

Since $\mathbf{A}$ depends on the UE's location and $\overline{\mathbf{B}}$ depends on the transmitter locations and the intensity function of the EDs, we can note that when the UE is located near to a transmitter, the beamformer looks like a transmitter selection process, i.e., the nearest transmitter's weight is dominant to the others. This 


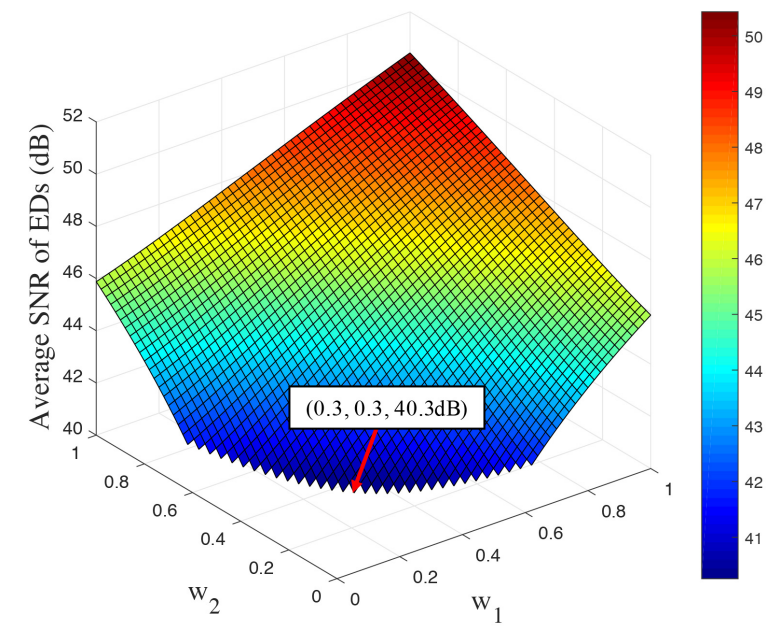

(a) The average SNR of EDs for a UE at $(0,1)$

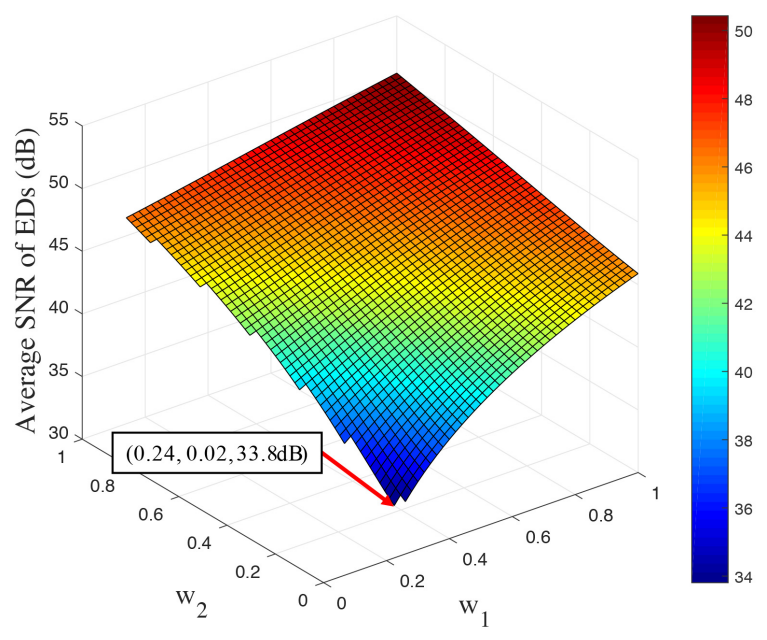

(b) The average SNR of EDs for a UE at $(2,1)$

Fig. 2. The average SNR of EDs for different UE locations plotted against beamforming weights. Two transmitters $T_{1}$ and $T_{2}$ are located at $(2.5,0)$ and $(-2.5,0)$, respectively. The room size is $L=8 \mathrm{~m}$ and $W=8 \mathrm{~m} . \rho_{U}=40 \mathrm{~dB}$ is applied.

is because the eigenvector corresponding to the maximum eigenvalue is significantly affected by the maximum diagonal element of $\mathbf{A}$ when the UE is near to the transmitter.

To illustrate this point, let us take an example of a simple VLC network where two transmitters are located at $T_{1}=$ $(2.5,0)$ and $T_{2}=(-2.5,0)$ in an $8 \times 8 \mathrm{~m}^{2}$ square room. The center of the room is located at the origin of our coordinate system. Figs. 2(a) and (b) show the average SNR of EDs according to a different set of beamformer weights when the UE is located at $(0,1)$ and $(2,1)$, respectively. The required SNR of the UE is $\rho_{U}=40 \mathrm{~dB}$. In Fig. 2(a), since the UE is located at the exact middle point of the two transmitters, the weight values for the two transmitters that minimize the average SNR of the EDs are equivalent, i.e., $\mathbf{w}^{*} \approx(0.3,0.3)$. However, when the UE is nearer to $T_{1}$ as in Fig. 2(b), we can see that the beamformer resembles LED selection. More specifically, $\mathbf{w}^{*} \approx(0.24,0.02)$ in this example. We can thus surmise that secrecy performance will be similar for

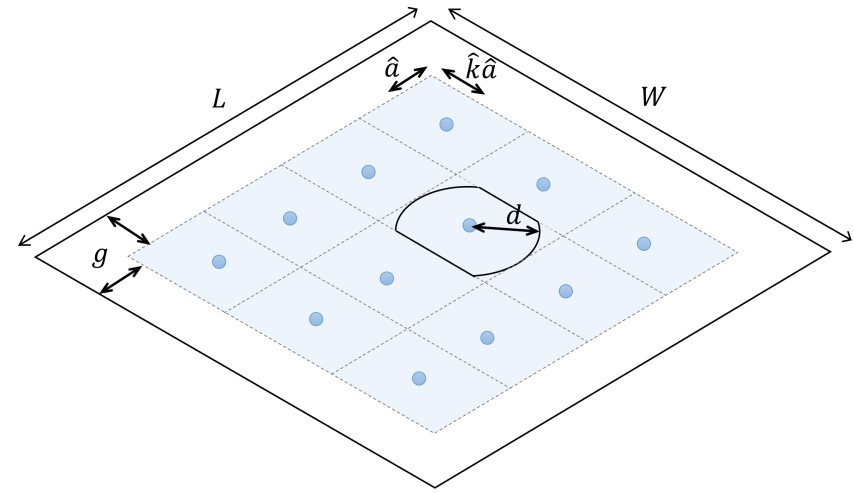

Fig. 3. The room configuration for LED selection.

beamforming and transmitter selection when the UE is located close to a transmitter ${ }^{5}$.

With regard to practicalities of implementation, the complexity of the beamforming scheme can be reasonably high due to the use of multiple transmitters. Even though we can efficiently find an eigenvector related to the maximum eigenvalue by using the power method or the Rayleigh quotient method, the computational complexity still can be significant since complexity grows with $N^{2}$. Additionally, it might not be practical to accurately estimate the intensity function that describes ED locations. These arguments motivate further investigation of the performance of LED selection in the context of VLC systems with randomly distributed EDs.

\section{LED SELECTION}

We turn our attention to the simple method of LED selection. In the LED selection scheme, the nearest transmitter to the UE is selected to transmit the information bearing signal. In this section, we first investigate the SNR and transmission rate performance metrics with LED selection. We then analyze the SOP. Closed-form expressions for the upper and lower bounds of the SOP with the LED selection are derived.

The room configuration in Fig. 3 is used for our analysis, where multiple transmitters are attached to the ceiling such that the coverage area is identical (but translated in the work plane) for each of them. The coverage area is defined as the area where a transmitter is responsible for supporting the UE. According to the LED selection scheme, the coverage area is determined by the distance between the transmitter and the UE; the nearest transmitter is responsible for supporting the UE. We assume that the UE can be located in the coverage $\operatorname{area}^{6}$, i.e., the shaded area, while multiple EDs can (randomly) position themselves anywhere in the entire area of the room. The parameters $\hat{a}$ and $\hat{k}$ in the figure denote the half length of the rectangular coverage area's width and the ratio of length to width of the coverage area. By defining the number of rows and columns of the LED arrangement as

\footnotetext{
${ }^{5}$ We will further explore the secrecy performance for both schemes later in Section V.

${ }^{6}$ One may think of this restriction as a policy instigated to guarantee the security of UE, through the restriction of the UE to a "safety zone". On the other hand, it is fairly easy to see that this model reflects many realistic scenarios.
} 
$N_{r}$ and $N_{c}$, respectively, the relations $\hat{a}=(L / 2-g) / N_{r}$ and $\hat{k}=(W / 2-g) /\left(N_{c} \hat{a}\right)$ can be deduced, where $g$ denotes the thickness of the edge zone. The UE and the EDs are assumed to be uniformly distributed according to homogeneous BPP (with one point) and a homogeneous $\operatorname{PPP} \Phi_{E}$ with intensity $\lambda_{E}$, respectively.

\section{A. SNR and Transmission Rate Analysis}

Since only the nearest transmitter is selected to transmit a signal to the UE, the vector representing LED selection $\mathbf{w}_{\mathbf{s}}$ can be described by

$$
\mathbf{w}_{\mathbf{s}}=\omega \mathbf{e}_{i}
$$

where $\omega$ is the weight of the selected transmitter and $\mathbf{e}_{i}$ is the $i$ th column of the identity matrix corresponding to the maximum diagonal element of $\mathbf{A}$, i.e., the $i$ th transmitter is the nearest. Therefore, the average SNR of the EDs when LED selection is employed is given by

$$
\bar{\gamma}_{E}=\varphi \mathbf{w}_{\mathbf{s}}^{\mathbf{T}} \overline{\mathbf{B}} \mathbf{w}_{\mathbf{s}} \text {. }
$$

If there exists a required SNR for the UE ( $\rho_{U}$, as in (15)), $\omega$ should satisfy $\omega^{2} A_{i, i}^{2} \geq \rho_{U}$, where $A_{i, i}$ denotes the maximum diagonal element of A. Similarly, the SNR of the UE can be described by

$$
\gamma_{U}=\varphi \mathbf{w}_{\mathbf{s}}^{\mathbf{T}} \mathbf{A} \mathbf{w}_{\mathbf{s}}
$$

Also, if there exists a constraint on the average SNR of the EDs $\left(\bar{\rho}_{E}\right.$, as in (22)), $\omega$ should satisfy $\omega^{2} \bar{B}_{i, i}^{2} \leq \bar{\rho}_{E}$, where $\bar{B}_{i, i}$ is the $i$ th diagonal element of $\overline{\mathbf{B}}$.

Moreover, the average upper bound on the transmission rate of the ED channel with LED selection can be written as

$$
\bar{C}_{E}^{\text {upper }}=\mathbb{E}\left[\frac{1}{2} \log \left(1+\varphi \omega^{2} h_{i, E_{e}}^{2}\right)\right]
$$

where $h_{i, E_{e}}$ is the channel gain from the $i$ th transmitter to eavesdropper $E_{e}$. Also, the lower bound on the transmission rate of the $\mathrm{UE}$ is given by

$$
C_{U}^{\text {lower }}=\frac{1}{2} \log \left(1+\frac{2 \varphi \mathbf{w}_{\mathbf{s}}^{\mathbf{T}} \mathbf{A} \mathbf{w}_{\mathbf{s}}}{\pi e}\right) .
$$

\section{B. Secrecy Outage Probability}

Here, we calculate upper and lower bounds on the SOP for the LED selection scheme. To this end, we necessarily must know something about the SNR statistics for the UE and the nearest ED, hence, the worst case ED. Thus, we begin by providing results for the probability density function (PDF) and the cumulative distribution function (CDF) for these random variables. To simplify the calculation of the SOP, we assume the weight of the selected transmitter's beamforming vector is always one. This assumption is justified since, as we will see, the beamforming weight itself does not significantly affect the SOP when LED selection is adopted.

Lemma 1. The CDF and PDF of the received UE SNR $\gamma_{U}$ are given by (35) and (36) at the top of the next page, respectively, where $\zeta=\left(\alpha^{2} I_{D C}^{2} K^{2}\right) / \sigma^{2}$, and $y_{i}$ and $K_{i}$ for $i \in\{1,2,3,4\}$ are given by

$$
\begin{aligned}
& y_{1}=\zeta\left(\hat{a}^{2}\left(1+\hat{k}^{2}\right)+Z^{2}\right)^{-3-m}, \\
& y_{2}=\zeta\left(\hat{a}^{2} \hat{k}^{2}+Z^{2}\right)^{-3-m}, \\
& y_{3}=\zeta\left(\hat{a}^{2}+Z^{2}\right)^{-3-m}, \\
& y_{4}=\zeta Z^{-2(3+m)}
\end{aligned}
$$

and

$$
\begin{aligned}
K_{1}= & \frac{\left(2 \sqrt{\hat{k}^{2}-1} / \hat{k}^{2}-2 \arccos \left(\frac{1}{\hat{k}}\right)\right)}{a(\hat{k}-1)} \\
K_{2}= & \pi-\hat{a} K_{1}, \\
K_{3}= & 2\left(\arccos \left(\frac{1}{\hat{k}}\right)-\arccos \left(\frac{1}{\sqrt{\hat{k}^{2}+1}}\right)-\arccos \left(\frac{\hat{k}}{\sqrt{\hat{k}^{2}+1}}\right)\right. \\
& \left.+\frac{2 \hat{k}}{\hat{k}^{2}+1}-\frac{\sqrt{\hat{k}^{2}-1}}{\hat{k}^{2}}\right) /\left(\hat{a}\left(\sqrt{\hat{k}^{2}+1}-\hat{k}\right)\right), \\
K_{4}= & \pi-2\left(\arccos \left(\frac{1}{\hat{k}}\right)-\frac{\sqrt{\hat{k}^{2}-1}}{\hat{k}^{2}}\right)-\hat{k} \hat{a} K_{3} .
\end{aligned}
$$

Proof. See Appendix A.

Lemma 2. The $C D F$ and $P D F$ of the received SNR for the nearest $E D$ relative to the selected transmitter $\gamma_{E}^{*}$ are given by

$$
\begin{aligned}
& F_{\gamma_{E}^{*}}(x)=e^{\lambda_{E} \pi\left(Z^{2}-\left(\frac{x}{\zeta}\right)^{-\frac{1}{m+3}}\right)}, \\
& f_{\gamma_{E}^{*}}(x)=\frac{\lambda_{E} \pi\left(\frac{x}{\zeta}\right)^{-\frac{1}{m+3}}}{x(m+3)} e^{\lambda_{E} \pi\left(\left(\frac{x}{\zeta}\right)^{-\frac{1}{m+3}}-Z^{2}\right)}
\end{aligned}
$$

for $0 \leq x \leq \zeta Z^{-2(m+3)}$.

Proof. See Appendix B.

According to (10), the upper and lower bounds of the SOP can be written as

$$
\begin{aligned}
P_{\mathrm{SO}}^{\text {upper }} & =\mathbb{P}\left(C_{s}^{\text {lower }} \leq C_{\mathrm{th}}\right) \\
& =\mathbb{P}\left(\frac{6 \gamma_{U}+3 \pi e}{\pi e \gamma_{E}^{*}+3 \pi e} \leq 2^{2 C_{\mathrm{th}}}\right) \\
& =\mathbb{P}\left(\gamma_{U} \leq a \gamma_{E}^{*}+3 a-\pi e / 2\right)
\end{aligned}
$$

and

$$
\begin{aligned}
P_{\text {SO }}^{\text {lower }} & =\mathbb{P}\left(C_{S}^{\text {upper }} \leq C_{\mathrm{th}}\right) \\
& =\mathbb{P}\left(\frac{\gamma_{U}+1}{\gamma_{E}^{*}+1} \leq 2^{2 C_{\mathrm{th}}}\right) \\
& =\mathbb{P}\left(\gamma_{U} \leq b \gamma_{E}^{*}+b-1\right)
\end{aligned}
$$

respectively, where $a=\pi e 2^{2 C_{\text {th }}} / 6$ and $b=2^{2 C_{\text {th }}}$. Thus, the upper and lower bounds on the SOP can be calculated by appropriately integrating over the PDFs of $\gamma_{U}$ and $\gamma_{E}^{*}$.

Firstly, the upper bound on the SOP can be calculated to 


$$
F_{\gamma_{U}}(y)=\left\{\begin{array}{lr}
1-\left(K_{4}\left((y / \zeta)^{\frac{-1}{m+3}}-Z^{2}\right)+K_{3}\left((y / \zeta)^{\frac{-1}{m+3}}-Z^{2}\right)^{3 / 2}\right) /\left(4 \hat{k} \hat{a}^{2}\right) & \text { for } \quad y_{1}<y \leq y_{2} \\
1-\left(K_{2}\left((y / \zeta)^{\frac{-1}{m+3}}-Z^{2}\right)+K_{1}\left((y / \zeta)^{\frac{-1}{m+3}}-Z^{2}\right)^{3 / 2}\right) /\left(4 \hat{k} \hat{a}^{2}\right) & \text { for } \quad y_{2}<y \leq y_{3} \\
1-\pi\left((y / \zeta)^{\frac{-1}{m+3}}-Z^{2}\right) /\left(4 \hat{k} \hat{a}^{2}\right) & \text { for } \quad y_{3}<y \leq y_{4}
\end{array}\right.
$$

$$
f_{\gamma_{U}}(y)= \begin{cases}(y / \zeta)^{-\frac{1}{m+3}}\left(3 K_{3} \sqrt{(y / \zeta)^{-\frac{1}{m+3}}-Z^{2}}+2 K_{4}\right) /\left(8 \hat{a}^{2} \hat{k}(m+3) y\right) & \text { for } \quad y_{1}<y \leq y_{2} \\ (y / \zeta)^{-\frac{1}{m+3}}\left(3 K_{1} \sqrt{(y / \zeta)^{-\frac{1}{m+3}}-Z^{2}}+2 K_{2}\right) /\left(8 \hat{a}^{2} \hat{k}(m+3) y\right) & \text { for } \quad y_{2}<y \leq y_{3} \\ \pi(y / \zeta)^{-\frac{1}{m+3}} /\left(4 \hat{a}^{2} \hat{k}(m+3) y\right) & \text { for } \quad y_{3}<y \leq y_{4}\end{cases}
$$

yield

$$
\begin{aligned}
P_{\mathrm{SO}}^{\text {upper }}= & \underbrace{\int_{y_{1}}^{y_{4}} \int_{\frac{y-3 a+\pi e / 2}{a}}^{\frac{y_{4}-3 a+\pi e / 2}{a}} f_{\gamma_{E}^{*}}(x) f_{\gamma_{U}}(y) \mathrm{d} x \mathrm{~d} y}_{U_{1}} \\
& +\underbrace{\int_{y_{1}}^{y_{4}} \int_{\frac{y_{4}-3 a+\pi e / 2}{a}}^{y_{4}} f_{\gamma_{E}^{*}}(x) f_{\gamma_{U}}(y) \mathrm{d} x \mathrm{~d} y}_{U_{2}} .
\end{aligned}
$$

Here, we ignore the $(-3 a+\pi e / 2) / a$ term in the integration limits, because it is small enough ${ }^{7}$ not to meaningfully affect our calculation. Thus, we calculate the first term $U_{1}$ to yield (43) at the top of the next page. Then, the closed-form expressions for $J_{1}, J_{2}$, and $J_{3}$ in (43) can be calculated by (2.325.6) in [29] according to (44a), (44b), and (44c). Finally, the second term $U_{2}$ in (42) can be written as

$$
U_{2} \approx F_{\gamma_{E}^{*}}\left(y_{4}\right)-F_{\gamma_{E}^{*}}\left(\frac{y_{4}}{a}\right) .
$$

The lower bound on the SOP can be calculated to yield

$$
\begin{aligned}
P_{\mathrm{SO}}^{\text {lower }}= & \int_{y_{1}}^{y_{4}} \int_{\frac{y-b+1}{b}}^{\frac{y_{4}-b+1}{b}} f_{\gamma_{E}^{*}}(x) f_{\gamma_{U}}(y) \mathrm{d} x \mathrm{~d} y \\
& +\int_{y_{1}}^{y_{4}} \int_{\frac{y_{4}-b+1}{b}}^{y_{4}} f_{\gamma_{E}^{*}}(x) f_{\gamma_{U}}(y) \mathrm{d} x \mathrm{~d} y .
\end{aligned}
$$

Here, we ignore the $(-b+1) / b$ term in a similar manner as in (42). Therefore, since (46) has an identical expression to (42), we have the closed-form of the lower bound on SOP by simply changing the variable $a$ to $b$ in the closed-form expression of the upper bound.

From the closed-form expressions for the upper and lower bounds on the SOP, we note that the SOP is inversely proportional to $4 \hat{a}^{2} \hat{k}$ (see (44)), which is the coverage area

\footnotetext{
${ }^{7}$ The absolute value of this term is less than 3 for $C_{\mathrm{th}}=1 \mathrm{bit} / \mathrm{Hz} / \mathrm{s}$, while $y_{1} / a$ is larger than $5 \times 10^{3}$ with the parameters used in Section V. The high SNR is obtainable in VLC system, which is an indirect consequence of the illumination requirements [28].
}

TABLE II. Simulation Parameters

\begin{tabular}{c|c}
\hline \multicolumn{2}{c}{ Room configuration } \\
\hline Length $(\mathrm{L}) \times$ Width $(\mathrm{W})$ & $10 \times 10 \mathrm{~m}^{2}$ \\
Height from the work plane $(\mathrm{Z})$ & $3 \mathrm{~m}$ \\
Number of light fixtures & 4 \\
Number of LEDs per fixture & 9 \\
\hline LED electrical and optical characteristics \\
\hline Average optical power per LED & $8 \mathrm{~W}$ \\
Optical power / current $\eta$ & 5 \\
Nominal half-intensity angle $\Phi_{1 / 2}$ & $60^{\circ}$ \\
Modulation index $\alpha$ & 0.5 \\
\hline \multicolumn{2}{c}{ Optical receiver characteristics } \\
\hline Photodetector's responsivity & $0.54 \mathrm{~mA} / \mathrm{mW}^{2}$ \\
Photodetector's physical area $A_{P D}$ & $1 \mathrm{~cm}{ }^{2}$ \\
Lens refractive index $\kappa$ & 1.5 \\
Noise power $\sigma^{2}$ & $-98.35 \mathrm{dBm}$ \\
\hline
\end{tabular}

of each transmitter. In other words, if the room size is fixed, then the SOP can be decreased by increasing the number of transmitters to reduce the coverage area.

\section{NUMERICAL RESUlTS}

In this section, we provide numerical results to verify our analysis. The room configuration and simulation parameters are provided in Table II. We use the Cartesian coordinate system to identify positions of transmitters and receivers, where the center of the room is located at the origin. We consider a VLC network where four transmitters are symmetrically located, i.e., their locations can be described by $\left( \pm d_{0}, \pm d_{0}\right)$. A homogeneous PPP describes the ED locations.

\section{A. Signal-to-Noise Ratio}

Figs. 4 and 5 show the comparison of the average SNR of EDs as a function of the UE location by using beamforming, LED selection and repetition coding schemes for the different locations of transmitters, where $\lambda_{E}=0.05$ and $\rho_{U}=20 \mathrm{~dB}$. Since four transmitters are symmetrically located, we only show the results for the UE locations within the first quadrant. 


$$
\begin{aligned}
& U_{1} \approx \int_{y_{1}}^{y_{4}}\left(F_{\gamma_{E}^{*}}\left(\frac{y_{4}}{a}\right)-F_{\gamma_{E}^{*}}\left(\frac{y}{a}\right)\right) f_{\gamma_{U}}(y) \mathrm{d} y=F_{\gamma_{E}^{*}}\left(\frac{y_{4}}{a}\right) \int_{y_{1}}^{y_{4}} f_{\gamma_{U}}(y) \mathrm{d} y-\int_{y_{1}}^{y_{4}} F_{\gamma_{E}^{*}}\left(\frac{y}{a}\right) f_{\gamma_{U}}(y) \mathrm{d} y \\
& =F_{\gamma_{E}^{*}}\left(\frac{y_{4}}{a}\right)-\underbrace{\int_{y_{1}}^{y_{2}} e^{\lambda_{E} \pi\left(Z^{2}-\left(\frac{y}{a \zeta}\right)^{-\frac{1}{m+3}}\right)} \frac{\left(\frac{y}{\zeta}\right)^{-\frac{1}{m+3}}\left(3 K_{3} \sqrt{\left(\frac{y}{\zeta}\right)^{-\frac{1}{m+3}}-Z^{2}}+2 K_{4}\right)}{8 \hat{a}^{2} \hat{k}(m+3) y} \mathrm{~d} y}_{J_{1}} \\
& -\underbrace{\int_{y_{2}}^{y_{3}} e^{\lambda_{E} \pi\left(Z^{2}-\left(\frac{y}{a \zeta}\right)^{-\frac{1}{m+3}}\right)} \underbrace{\left(\frac{y}{\zeta}\right)^{-\frac{1}{m+3}}\left(3 K_{1} \sqrt{\left(\frac{y}{\zeta}\right)^{-\frac{1}{m+3}}-Z^{2}}+2 K_{2}\right)}_{J_{3}} d y}_{J_{2}}-\underbrace{\int_{y_{3}}^{y_{4}} e^{\lambda_{E} \pi\left(Z^{2}-\left(\frac{y}{a \zeta}\right)^{-\frac{1}{m+3}}\right)} \frac{\pi\left(\frac{y}{\zeta}\right)^{-\frac{1}{m+3}}}{4 \hat{a}^{2} \hat{k}(m+3) y} \mathrm{~d} y}_{\hat{a}^{2} \hat{k}(m+3) y}
\end{aligned}
$$

$$
\begin{aligned}
& J_{1}=\frac{1}{8 \hat{a}^{2} \hat{k}}\left(\frac{2 a^{\frac{-1}{m+3}} e^{\lambda_{E} \pi Z^{2}}}{\lambda_{E} \pi}\left(e^{-\lambda_{E} \pi\left(\frac{y_{2}}{a \zeta}\right)^{\frac{-1}{m+3}}}-e^{-\lambda_{E} \pi\left(\frac{y_{1}}{a \zeta}\right)^{\frac{-1}{m+3}}}\right) K_{4}+\frac{\left.3 e^{-\lambda_{E} \pi Z^{2}\left(-1+a \frac{1}{m+3}\right.}\right) K_{3}}{2 \pi^{3 / 2}}\right. \\
& \left.\cdot \sum_{i \in\{1,2\}}(-1)^{i+1}\left(\frac{\left(\left(\frac{y_{i}}{\zeta}\right)^{\frac{-1}{m+3}}-Z^{2}\right)^{3 / 2}\left(\sqrt{\pi}-2 \Gamma\left(\frac{3}{2}, a^{\frac{1}{m+3}} \lambda_{E} \pi\left(\left(\frac{y_{i}}{\zeta}\right)^{\frac{-1}{m+3}}-Z^{2}\right)\right)\right)}{\left(a^{\frac{1}{m+3}} \lambda_{E}\left(\left(\frac{y_{i}}{\zeta}\right)^{\frac{-1}{m+3}}-Z^{2}\right)\right)^{3 / 2}}\right)\right) \\
& J_{2}=\frac{1}{8 \hat{a}^{2} \hat{k}}\left(\frac{2 a^{\frac{-1}{m+3}} e^{\lambda_{E} \pi Z^{2}}}{\lambda_{E} \pi}\left(e^{-\lambda_{E} \pi\left(\frac{y_{3}}{a \zeta}\right)^{\frac{-1}{m+3}}}-e^{-\lambda_{E} \pi\left(\frac{y_{2}}{a \zeta}\right)^{\frac{-1}{m+3}}}\right) K_{2}+\frac{\left.3 e^{-\lambda_{E} \pi Z^{2}\left(-1+a^{\frac{1}{m+3}}\right.}\right) K_{1}}{2 \pi^{3 / 2}}\right. \\
& \left.\sum_{i \in\{2,3\}}(-1)^{i}\left(\frac{\left(\left(\frac{y_{i}}{\zeta}\right)^{\frac{-1}{m+3}}-Z^{2}\right)^{3 / 2}\left(\sqrt{\pi}-2 \Gamma\left(\frac{3}{2}, a^{\frac{1}{m+3}} \lambda_{E} \pi\left(\left(\frac{y_{i}}{\zeta}\right)^{\frac{-1}{m+3}}-Z^{2}\right)\right)\right)}{\left(a^{\frac{1}{m+3}} \lambda_{E}\left(\left(\frac{y_{i}}{\zeta}\right)^{\frac{-1}{m+3}}-Z^{2}\right)\right)^{3 / 2}}\right)\right) \\
& J_{3}=\frac{1}{4 \hat{a}^{2} \hat{k} \lambda_{E}}\left(a^{\frac{-1}{m+3}} e^{\lambda_{E} \pi Z^{2}}\left(e^{-\lambda_{E} \pi\left(\frac{y_{4}}{a \zeta}\right)^{\frac{-1}{m+3}}}-e^{-\lambda_{E} \pi\left(\frac{y_{3}}{a \zeta}\right)^{\frac{-1}{m+3}}}\right)\right)
\end{aligned}
$$

The repetition coding scheme [30] is given as a benchmark in which all of the precoding weights are set to one, i.e., $\mathbf{w}=\mathbb{1}$, since there is only one active UE at a time in the considered configuration. In addition, for a fair comparison, we scale down the modulation index $\alpha$ such that the average SNR of EDs with using the repetition coding meets $\rho_{U}$.

All of the bottom surfaces in (a) and (b) of Figs. 4 and 5 denote the average SNR of EDs as a function of the UE location when using the beamforming, and the top surfaces in (a) and (b) of Figs. 4 and 5 denote the results for the LED selection and the repetition coding, respectively. Firstly, when four transmitters are located closely to each other in the center area of the room as in Fig. 4, the transmitters emit a signal with high power to the UE that is located in the outer area to satisfy $\rho_{U}$. Due to the broadcasting characteristic of light, the EDs can eavesdrop the signal easily; thus their average SNR also increases as shown in Figs. 4(a) and 4(b). In this case, the EDs can achieve a higher average SNR than $\rho_{U}$ if the UE is away from the transmitter. On the contrary, when the distance between the transmitter and the UE decreases, one can see that the average SNR of the EDs also decreases because of the decrease in transmitted power. On the other hand, we see that when the four transmitters are adequately separated from each other as in Fig. 5, both beamforming and LED selection exploit the spatial advantage to decrease the average SNR of EDs. The average SNR of EDs is less than $\rho_{U}$ for most of the area except for around the center point of the transmitters, i.e., near $(0,0)$.

Furthermore, in Figs. 4(c) and 5(c), the ratio of the average SNRs of EDs when using the LED selection and the beamforming is given as the bottom surface, and the ratio of the average SNRs of EDs when using the repetition coding and the beamforming is given as the top surface. We can easily note that the average SNR of EDs with repetition coding is much higher than the beamforming in both configurations. In contrary, the ratio of the average SNRs of EDs of LED selection and beamforming is small when the UE is located near to the transmitter, while beamforming can slightly outperform 




(a) The average SNR of EDs with beamforming and LED selection

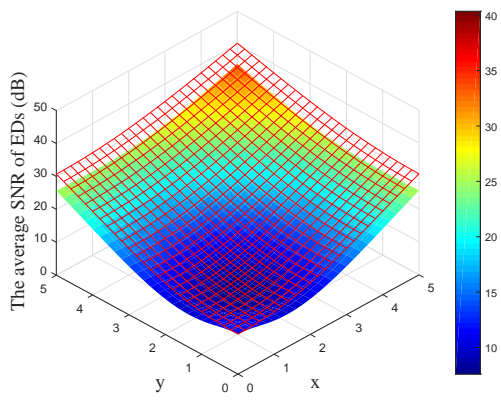

(b) The average SNR of EDs with beamforming and repetition coding

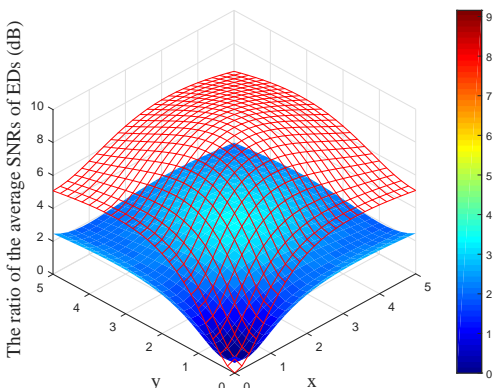

(c) The ratio of the average SNRs of EDs generalized by beamforming

Fig. 4. The average SNR of EDs as a function of the UE location. The bottom surfaces in (a) and (b) denote the results for the beamforming (identical). The top surfaces denote LED selection and repetition coding, respectively. In (c), the ratio of the average SNRs of EDs are given in which the top surface denotes the ratio of repetition coding and beamforming, and the bottom surface denotes the ratio of LED selection and beamforming, respectively. Four transmitters are located at $( \pm 1, \pm 1)$. The numerical result for the locations of the UE within the 1 st quadrant are given. The intensity of the ED process is $\lambda_{E}=0.05$ and the required SNR of the UE is $\rho_{U}=20 \mathrm{~dB}$.

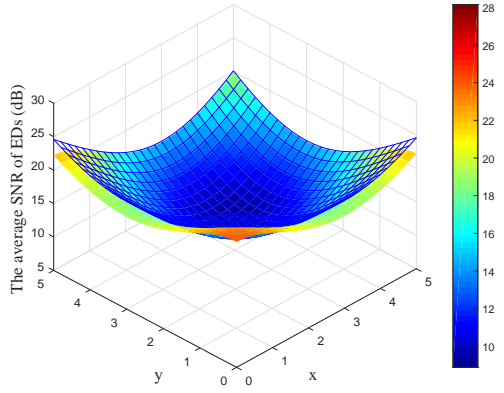

(a) The average SNR of EDs with beamforming and LED selection

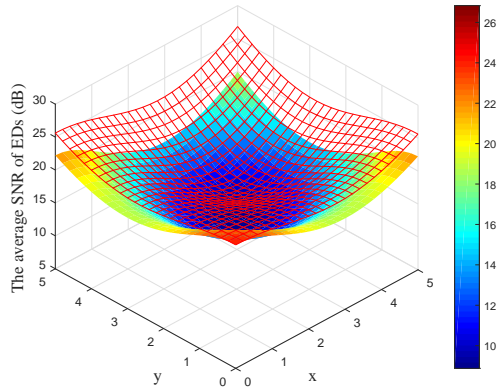

(b) The average SNR of EDs with beamforming and repetition coding

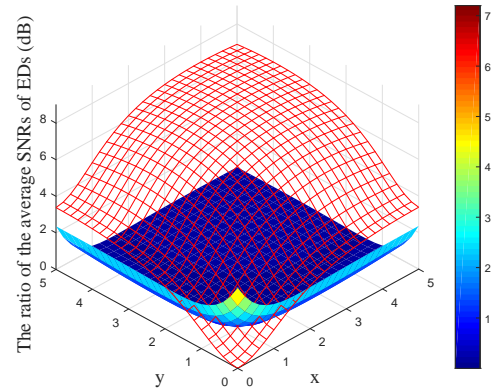

(c) The ratio of the average SNRs of EDs generalized by beamforming

Fig. 5. The average SNR of EDs as a function of the UE location. The bottom surfaces in (a) and (b) denote the results for the beamforming (identical). The top surfaces denote LED selection and repetition coding, respectively. In (c), the ratio of the average SNRs of EDs are given in which the top surface denotes the ratio of repetition coding and beamforming, and the bottom surface denotes the ratio of LED selection and beamforming, respectively. Four transmitters are located at $( \pm 3, \pm 3)$. The numerical result for the locations of the UE within the 1 st quadrant are given. The intensity of the ED process is $\lambda_{E}=0.05$ and the required SNR of the UE is $\rho_{U}=20 \mathrm{~dB}$.

LED selection when the UE is away from the transmitter.

Figs. 6 and 7 show a comparison of the SNR of the UE as a function of the UE location using beamforming, LED selection, repetition coding schemes for different locations of transmitters, where $\lambda_{E}=0.05$ and $\bar{\rho}_{E}=30 \mathrm{~dB}$. All of the top surfaces in (a) and (b) of Figs. 6 and 7 denote the SNR of the $\mathrm{UE}$ as a function of the UE location when using beamforming, and the bottom surfaces in (a) and (b) denote the results for the LED selection and the repetition coding, respectively. Firstly, when the transmitters are located closely together as in Fig. 6, the SNR of the UE can be high when the UE is located near to the transmitter. However, when the UE moves away from the transmitter, it is difficult for the UE to achieve an SNR higher than $\bar{\rho}_{E}$. This is because the transmitter should convey the signal with high enough power to reach the UE, which enables the EDs to overhear the signal easily. In contrast, when the transmitters are sufficiently separated as in Fig. 7, we note that the UE can achieve a higher SNR than $\bar{\rho}_{E}$ over almost the entire area of the room except for at the center point of the room, i.e., near $(0,0)$, as shown in Fig. $7($ a). This can be possible by selectively transmitting a signal to the UE without excessively increasing the signal power of other transmitters, thus exploiting the spatial benefit.

In addition, in Figs. 6(c) and 7(c), the ratio of the SNRs of the UE when using beamforming and LED selection is given as the bottom surface, and the ratio of the SNRs of the UE when using beamforming and repetition coding is given as the top surface. First, we can note that beamforming outperforms much more than the repetition coding in both configurations. These results show that the beamforming effectively transmits a signal to the UE trying not to expose the signal to EDs by taking the density of ED locations into account for the beamforming weights. In addition, we see from Fig. 6(c) that the ratio of the SNRs of the UE for beamforming and LED selection is not significant for the UE located near the transmitter, but it slightly increases as the distance between the transmitter and the UE grows. Similarly, from Fig. 7(c), we note that the ratio of the SNRs of the UE for beamforming and LED selection is not significant when the UE is close to the transmitter. Therefore, when the transmitters are adequately 


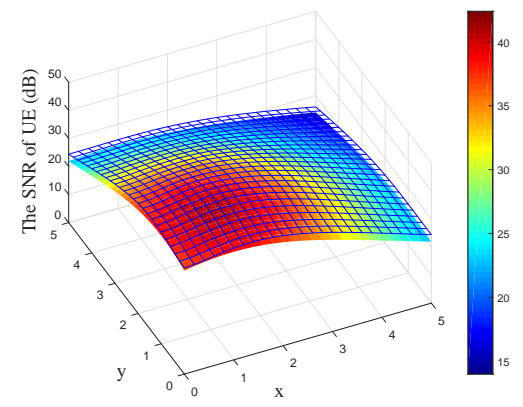

(a) The SNR of UE with beamforming and LED selection



(b) The SNR of UE with beamforming and repetition coding

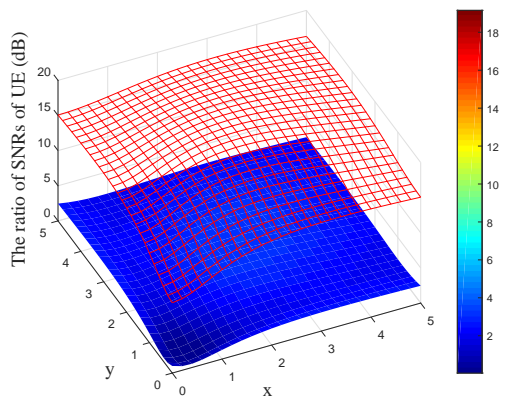

(c) The ratio of the SNRs of UE generalized by beamforming

Fig. 6. The average SNR of the UE as a function of the UE locations. The top surfaces in (a) and (b) denote the result for the beamforming (identical). The bottom surfaces denote LED selection and repetition coding, respectively. In (c), the ratio of the SNRs of UE are given in which the top surface denotes the ratio of beamforming and LED selection, and the bottom surface denotes the ratio of beamforming and repetition coding, respectively. Four transmitters are located at $( \pm 1, \pm 1)$. The numerical results for the locations of the UE within the 1st quadrant are given. The density of the ED process is $\lambda_{E}=0.05$ and the constraint on the average SNR of EDs is $\bar{\rho}_{E}=30 \mathrm{~dB}$.

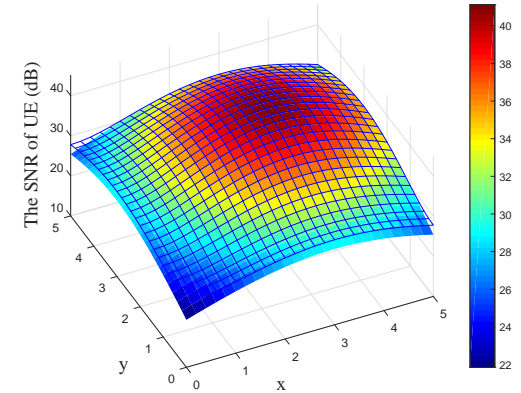

(a) The SNR of UE with beamforming and LED selection

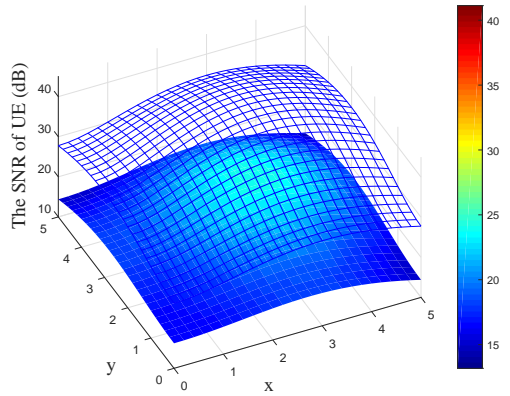

(b) The SNR of UE with beamforming and repetition coding

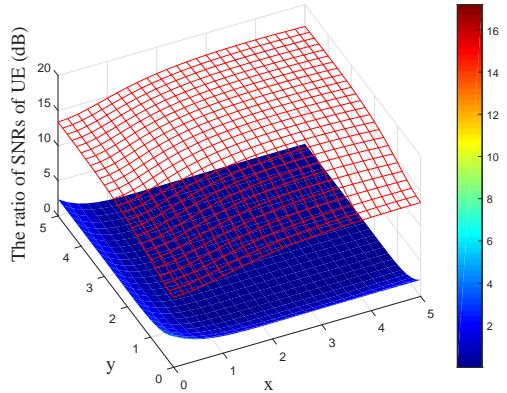

(c) The ratio of the SNRs of UE generalized by beamforming

Fig. 7. The average SNR of the UE as a function of the UE locations. The top surfaces in (a) and (b) denote the result for the beamforming (identical). The bottom surfaces denote LED selection and repetition coding, respectively. In (c), the ratio of the SNRs of UE are given in which the top surface denotes the ratio of beamforming and LED selection, and the bottom surface denotes the ratio of beamforming and repetition coding, respectively. Four transmitters are located at $( \pm 3, \pm 3)$. The numerical results for the locations of the UE within the 1 st quadrant are given. The density of the ED process is $\lambda_{E}=0.05$ and the constraint on the average SNR of EDs is $\bar{\rho}_{E}=30 \mathrm{~dB}$.

separated, and the UE is near to the transmitters, it is shown that the difference in secrecy performance between beamforming and LED selection is small. Therefore, considering the computational complexity and feasibility of beamforming, we conclude that LED selection may be an attractive option in some scenarios.

\section{B. Secrecy Outage Probability}

Fig. 8 shows the upper and lower bounds on the SOP with LED selection for different intensities of EDs and different values of $C_{\text {th }}$, where $N=4 \times 4, g=1 \mathrm{~m}, \hat{a}=1 \mathrm{~m}, \hat{k}=$ $1.25, L=10 \mathrm{~m}$, and $W=12 \mathrm{~m}$. Both simulated results and theoretical results are presented, which are shown to perfectly match. As can be seen from the figure, both the SOP upper and lower bounds increase as $\lambda_{E}$ and $C_{\text {th }}$ increase, as expected.

Fig. 9 shows a comparison of the upper bound on SOP among beamforming, LED selection and repetition coding for different numbers of transmitters and different values of $\lambda_{E}$, where $C_{\mathrm{th}}=0.5 \mathrm{bit} / \mathrm{Hz} / \mathrm{s}, g=1 \mathrm{~m}, L=10 \mathrm{~m}$, and $W=12 \mathrm{~m}$. It can be seen that as the number of transmitters increases, the upper bound on SOP decreases with beamforming and LED selection, while it increases with repetition coding. In the proposed beamforming and LED selection, the multiple spatially distributed transmitters efficiently decrease the possibility of eavesdropping by transmitting the legitimate data favorably to UE. In contrast, in the repetition coding, all of the transmitters emit the legitimate data with an equal power regardless of the location of the UE although the transmitters far away from the UE cannot largely increase the SNR of the UE. Thus, the advantage of repetition coding for EDs is much larger than that of the UE, then the SOP increases. Furthermore, when the number of transmitters is small, i.e., $N=2 \times 2$, the difference in the SOP for beamforming and LED selection is small. However, when $N$ is large, we can see that the upper bound on the SOP with beamforming is less than for LED selection. Since a large number of transmitters with beamforming can exploit the excessive spatial degrees of freedom to steer the signal toward the UE, the transmitters can significantly increase the SNR of the UE while suppressing the signal everywhere else inside the room. However, we 


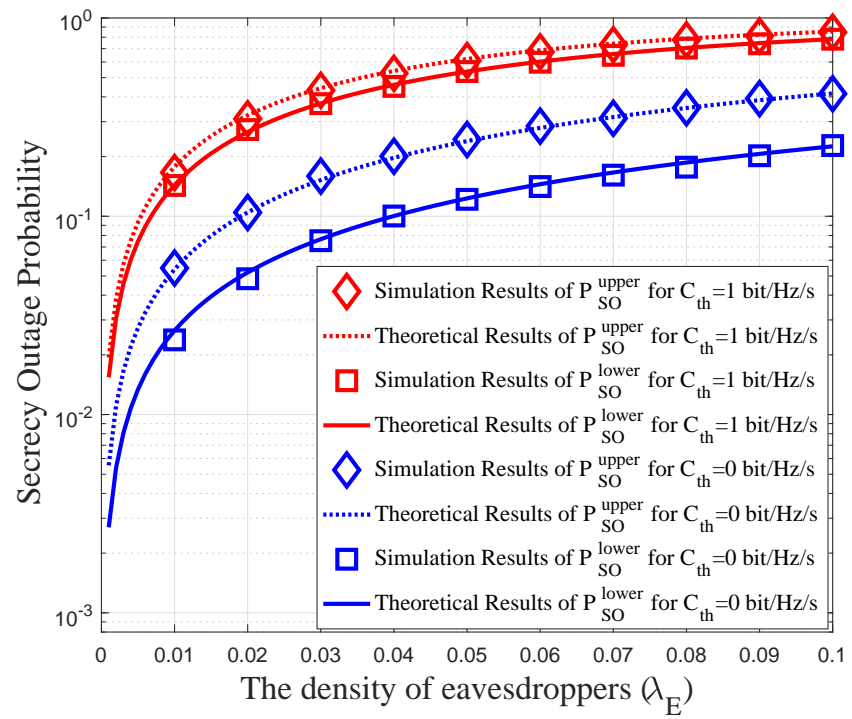

Fig. 8. The upper and lower bounds on the SOP with LED selection for different $C_{\text {th }}$ according to different intensities of EDs $\lambda_{E}$, where $N=4 \times 4, g=1 \mathrm{~m}, \hat{a}=1 \mathrm{~m}, \hat{k}=1.25, L=10 \mathrm{~m}$, and $W=12 \mathrm{~m}$.

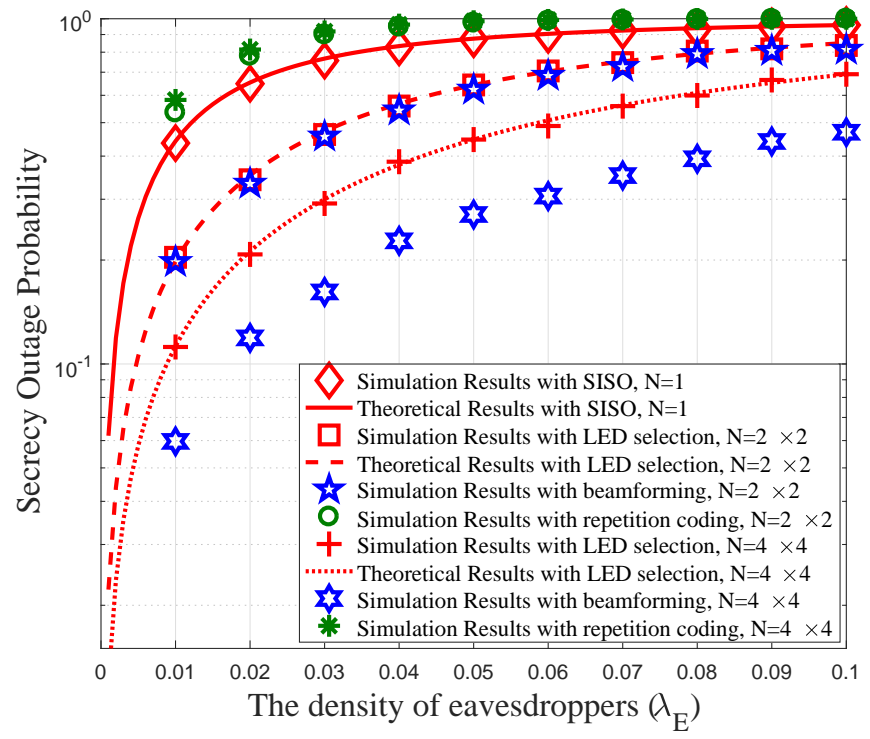

Fig. 9. A comparison of the upper bound on the SOP between LED selection and beamforming for different numbers of transmitters according to different intensities of EDs $\lambda_{E}$, where $C_{\text {th }}=0.5$ bit/Hz/s, $g=1 \mathrm{~m}, L=10 \mathrm{~m}$, and $W=12 \mathrm{~m}$.

need to consider that finding the maximum eigenvalue and its associated eigenvector with a large number of transmitters requires high computation complexity, which increases proportionally to $N^{2}$. However, it is worthwhile to mention that the proposed beamforming is still less complicated than the previous beamforming scheme proposed in [11], which has been shown to be NP-hard [31]. In our proposed beamforming, we have a deterministic way, i.e., the eigenvector of $\overline{\mathbf{B}}^{-1} \mathbf{A}$, to find the beamformer when (17) is satisfied.



Fig. 10. An example of $\hat{D}(d)$ for $\hat{a}=4 \mathrm{~m}, \hat{k}=1.5$. Note $\hat{a} \hat{k}=6 \mathrm{~m}$ and $\hat{a} \sqrt{\hat{k}^{2}+1}=7.21 \mathrm{~m}$. $\hat{a} \hat{k}=6 \mathrm{~m}$ and $\hat{a} \sqrt{\hat{k}^{2}+1}=7.21 \mathrm{~m}$

\section{CONCLUSION}

In this paper, we studied beamforming schemes and a simpler LED selection scheme to enhance the secrecy performance in VLC systems when multiple EDs are randomly distributed throughout the communication region. By using the beamforming scheme, we can minimize the average SNR of EDs (or indeed the worst case SNR of EDs) and maximize the SNR of the UE with only statistical information about ED locations. The LED selection scheme is not superior to the beamformer in the respect of secrecy performance; however, when the UE is located near to one of the transmitters, LED selection provides a good practical solution to enhancing secrecy performance without high computational complexity. Based on LED selection, closed-form approximations for the upper and lower bounds on the SOP were derived. Our results provide useful insight and analytic tools that can be used to enhance the secrecy in VLC systems and give a solid basis for further study.

\section{APPENDIX A PROOF OF THE LEMMA 1}

The UE is randomly located according to a homogeneous BPP in the shaded area in Fig. 3. Therefore, the distance in the work plane between the transmitter and the UE $d_{U}$ cannot exceed $\hat{a} \sqrt{\hat{k}^{2}+1}$. Thus, the CDF of $d_{U}$ is given by

$$
F_{d_{U}}(d)=\frac{A(d)}{4 \hat{k} \hat{a}^{2}} \quad \text { for } \quad 0<d \leq \hat{a} \sqrt{\hat{k}^{2}+1}
$$

where $A(d)$ denotes the area of the circle bounded by the rectangle as shown in Fig. 3. When the radius of the circular area where the UE locates, i.e., $d$, equals to $\hat{a}$, its circumference hits the small rectangle. Also, if $d$ increases further, the circular area starts to be bounded by the rectangle since the UE cannot exist out of the bounded circular area. If the UE locates out of the bounded circular area, the UE would 


$$
A(d)= \begin{cases}\pi d^{2} & \text { for } \quad 0<d \leq \hat{a} \\ \pi d^{2}-2\left(d^{2} \arccos \left(\frac{\hat{a}}{d}\right)-\hat{a} \sqrt{d^{2}-\hat{a}^{2}}\right) & \text { for } \quad \hat{a}<d \leq \hat{k} \hat{a} \\ \pi d^{2}-2\left(d^{2} \arccos \left(\frac{\hat{a}}{d}\right)-\hat{a} \sqrt{d^{2}-\hat{a}^{2}}\right)-2\left(d^{2} \arccos \left(\frac{\hat{a} \hat{k}}{d}\right)-\hat{a} \hat{k} \sqrt{d^{2}-(\hat{a} \hat{k})^{2}}\right) & \text { for } \hat{k} \hat{a}<d \leq \hat{a} \sqrt{\hat{k}^{2}+1}\end{cases}
$$

select another nearest transmitter. $A(d)$ is described as (48) at the top of this page.

Thus, $A(d)$ can be described as $A(d)=D(d) \cdot d^{2}$, where $D(d)$ can be approximated by applying a piecewise approximation with a linear function of $d$, i.e.,

$$
\hat{D}(d)= \begin{cases}\pi & \text { for } \quad 0<d \leq \hat{a} \\ K_{1} d+K_{2} & \text { for } \quad \hat{a}<d \leq \hat{k} \hat{a} \\ K_{3} d+K_{4} & \text { for } \quad \hat{k} \hat{a}<d \leq \hat{a} \sqrt{\hat{k}^{2}+1}\end{cases}
$$

To find the optimal $K_{i}$ for $i \in\{1,2,3,4\}$, we evaluate three coordinates $D(\hat{a}), D(\hat{a} \hat{k})$, and $D\left(\hat{a} \sqrt{\hat{k}^{2}+1}\right)$ as shown in Fig. 10. Using these values, we can easily calculate the approximation constants as shown in (38).

Finally, from (3) and (5a), the SNR of the UE can be described as a function of $d$ according to

$$
\gamma_{U}(d)=\frac{\alpha^{2} I_{D C}^{2} K^{2}\left(d^{2}+Z^{2}\right)^{-(m+3)}}{\sigma^{2}}=\zeta\left(d^{2}+Z^{2}\right)^{-(m+3)}
$$

where $\zeta=\left(\alpha^{2} I_{D C}^{2} K^{2}\right) / \sigma^{2}$. Thus, the CDF and PDF of $\gamma_{U}$ can be written as (35) and (36), respectively.

\section{APPENDIX B}

\section{PROOF OF THE LEMMA 2}

Since the EDs are randomly distributed according to a homogeneous PPP $\Phi_{E}$ with intensity $\lambda_{E}$ on the work plane, the PDF of the minimum distance in the work plane between the selected transmitter and the nearest ED, i.e., $d_{E}^{*}=\min _{e \in \Phi_{E}} d_{E_{e}}$, where $d_{E_{e}}$ is the distance in the work plane between the selected transmitter and $E_{e}$, is given by

$$
F_{d_{E}^{*}}(d)=1-\exp \left(-\lambda_{E} \pi d^{2}\right)
$$

for $0 \leq d \leq \infty$. This follows from contact distance distribution to the nearest point of the PPP [32]. Here, the unbounded upper limit implies that the number of EDs can be zero. The PDF of $d_{E}^{*}$ can be calculated as $f_{d_{E}^{*}}(d)=2 \lambda_{E} \pi d \exp \left(-\lambda_{E} \pi d^{2}\right)$ for $0 \leq d \leq \infty$. Therefore, since $\gamma_{E}^{*}$ also has the same relation with $d_{E}^{*}$ as (50), the PDF and CDF of $\gamma_{E}^{*}$ can be calculated as below.

$$
\begin{aligned}
f_{\gamma_{E}^{*}}(x) & =\frac{\lambda_{E} \pi\left(\frac{x}{\zeta}\right)^{-\frac{1}{m+3}}}{x(m+3)} e^{-\lambda_{E} \pi\left(-Z^{2}+\left(\frac{x}{\zeta}\right)^{-\frac{1}{m+3}}\right)}, \\
F_{\gamma_{E}^{*}}(x) & =\int_{0}^{x} f_{\gamma_{E}^{*}}(u) \mathrm{d} u \\
& =\int_{0}^{x} \frac{\lambda_{E} \pi\left(\frac{u}{\zeta}\right)^{-\frac{1}{m+3}}}{u(m+3)} e^{-\lambda_{E} \pi\left(-Z^{2}+\left(\frac{u}{\zeta}\right)^{-\frac{1}{m+3}}\right)} \mathrm{d} u
\end{aligned}
$$

$$
\begin{aligned}
& =\int_{\left(\frac{x}{\zeta}\right)^{-\frac{1}{m+3}}}^{\infty} \lambda_{E} \pi e^{-\lambda_{E} \pi\left(v-Z^{2}\right)} \mathrm{d} v \\
& =e^{\lambda_{E} \pi\left(Z^{2}-\left(\frac{x}{\zeta}\right)^{-\frac{1}{m+3}}\right)}
\end{aligned}
$$

for $0 \leq x \leq \zeta Z^{-2(m+3)}$, respectively, where $v=\left(\frac{u}{\zeta}\right)^{-\frac{1}{m+3}}$.

\section{REFERENCES}

[1] H. Haas, L. Yin, Y. Wang, and C. Chen, "What is LiFi?" J. Lightw. Technol., vol. 34, no. 6, pp. 1533-1544, Mar. 2016.

[2] J. G. Andrews, S. Buzzi, W. Choi, S. V. Hanly, A. Lozano, A. C. K. Soong, and J. C. Zhang, "What will 5G be?" IEEE J. Sel. Areas Commun., vol. 32, no. 6, pp. 1065-1082, Jun. 2014

[3] A. D. Wyner, "The wire-tap channel," Bell Syst. Tech. J., vol. 54, pp. 1355-1387, Jan. 1975.

[4] M. Bloch and J. Barros, Physical-Layer Security: From Information Theory to Security Engineering. Cambridge University Press, 2011.

[5] M. Haenggi, "The secrecy graph and some of its properties," in IEEE ISIT in Toronto, Canada, Jul. 2008, pp. 539-543.

[6] P. C. Pinto, J. Barros, and M. Z. Win, "Physical-layer security in stochastic wireless networks," in IEEE ICCS in Guangzhou, China, Nov. 2008, pp. 974-979.

[7] X. Zhou, R. K. Ganti, J. G. Andrews, and A. Hjorungnes, "On the throughput cost of physical layer security in decentralized wireless networks," Trans. Wireless Commun., vol. 10, no. 8, pp. 2764-2775, Aug. 2011.

[8] G. Geraci, S. Singh, J. G. Andrews, J. Yuan, and I. B. Collings, "Secrecy rates in broadcast channels with confidential messages and external eavesdroppers," IEEE Trans. Wireless Commun., vol. 13, no. 5, pp. 2931-2943, May 2014.

[9] T. X. Zheng, H. M. Wang, and Q. Yin, "On transmission secrecy outage of a multi-antenna system with randomly located eavesdroppers," IEEE Commun. Lett., vol. 18, no. 8, pp. 1299-1302, Aug. 2014.

[10] G. Chen, J. P. Coon, and M. D. Renzo, "Secrecy outage analysis for downlink transmissions in the presence of randomly located eavesdroppers," IEEE Trans. Inf. Forens. Security, vol. 12, no. 5, pp. 1195-1206, May 2017.

[11] A. Mostafa and L. Lampe, "Physical-layer security for MISO visible light communication channels," IEEE J. Sel. Areas Commun., vol. 33 , no. 9, pp. 1806-1818, Sep. 2015.

[12] —_ "Physical-layer security for indoor visible light communications," in IEEE ICC in Sydney, Australia, Jun. 2014, pp. 3342-3347.

[13] — "Securing visible light communications via friendly jamming," in IEEE Globecom Workshops in Austin, USA, Dec. 2014, pp. 524-529.

[14] H. Zaid, Z. Rezki, A. Chaaban, and M. S. Alouini, "Improved achievable secrecy rate of visible light communication with cooperative jamming," in IEEE GlobalSIP in Orlando, USA, Dec. 2015, pp. 1165-1169.

[15] M. A. Arfaoui, Z. Rezki, A. Ghrayeb, and M. S. Alouini, "On the secrecy capacity of MISO visible light communication channels," in IEEE Globecom in Washington D.C., USA, Dec. 2016, pp. 1-7.

[16] S. Cho, G. Chen, and J. P. Coon, "Secrecy analysis in visible light communication systems with randomly located eavesdroppers," in 2017 IEEE ICC Workshops in Paris, France, May 2017, pp. 475-480.

[17] T. Komine and M. Nakagawa, "Fundamental analysis for visible-light communication system using LED lights," IEEE Trans. Consum. Electron., vol. 50, no. 1, pp. 100-107, Feb. 2004.

[18] C. Chen, D. Basnayaka, and H. Haas, "Downlink SINR statistics in OFDM-based optical attocell networks with a poisson point process network model," in IEEE GLOBECOM, Dec. 2015, pp. 1-6. 
[19] A. Khisti and G. W. Wornell, "Secure transmission with multiple antennas I: The MISOME wiretap channel," IEEE Trans. Inf. Theory, vol. 56, no. 7, pp. 3088-3104, Jul. 2010.

[20] A. Lapidoth, S. M. Moser, and M. A. Wigger, "On the capacity of free-space optical intensity channels," IEEE Trans. Inf. Theory, vol. 55, no. 10 , pp. $4449-4461$, Oct. 2009.

[21] O. Ozel, E. Ekrem, and S. Ulukus, "Gaussian wiretap channel with an amplitude constraint," in IEEE Inf. Theory Workshop in Lausanne, Switzerland, Sep. 2012, pp. 5553-5563.

[22] S. M. Moser, M. Mylonakis, L. Wang, and M. Wigger, "Asymptotic capacity results for MIMO wireless optical communication," in IEEE ISIT, Jun. 2017, pp. 536-540.

[23] I. Krikidis, J. S. Thompson, and S. Mclaughlin, "Relay selection for secure cooperative networks with jamming," IEEE Trans. Wireless Commun., vol. 8, no. 10, pp. 5003-5011, Oct. 2009.

[24] H. Hui, A. L. Swindlehurst, G. Li, and J. Liang, "Secure relay and jammer selection for physical layer security," IEEE Signal Process. Lett., vol. 22, no. 8, pp. 1147-1151, Aug. 2015.

[25] G. Chen, Y. Gong, P. Xiao, and J. A. Chambers, "Dual antenna selection in secure cognitive radio networks," IEEE Trans. Veh. Technol., vol. 65 , no. 10, pp. 7993-8002, Oct. 2016.

[26] S. Boyd and L. Vandenberghe, Convex Optimization. New York, NY, USA: Cambridge University Press, 2004

[27] T. Lipp and S. Boyd, "Variations and extension of the convex-concave procedure," Optimization and Engineering, vol. 17, no. 2, pp. 263-287, Jun. 2016

[28] J. Grubor, S. Randel, K. D. Langer, and J. W. Walewski, "Bandwidthefficient indoor optical wireless communications with white lightemitting diodes," in 6th CSNDSP in Graz, Austria, Jul. 2008, pp. 165169.

[29] I. S. Gradshteyn and I. M. Ryzhik, Table of integrals, series, and products, 7th ed. Elsevier/Academic Press, 2007.

[30] D. Tse and P. Viswanath, Fundamentals of Wireless Communication New York, NY, USA: Cambridge University Press, 2005.

[31] E. Wong, "Active-set methods for quadratic programming," Ph.D. dissertation, Department of Mathematics, University of California, San Diego, June 2011.

[32] A. Baddeley, E. Rubak, and R. Turner, Spatial point patterns: methodology and applications with R. CRC Press, 2015.

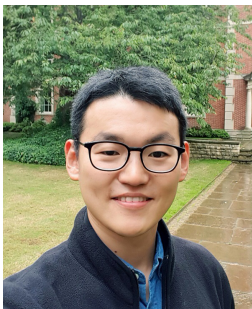

Sunghwan Cho (S'17) received the B.S. degree (summa cum laude) in electrical engineering from the Korea Military Academy, South Korea, in 2007. He earned his M.S. degree in communications from the Georgia Institute of Technology, USA, in 2011. From 2016, he is a DPhil student in the Department of Engineering Science at the University of Oxford, U.K., under the supervision of Professor Justin P. Coon. He has worked as an army officer of the Republic of Korea Army, currently holding the rank of a major. His current research interests include physical layer security, stochastic geometry, and visible light communications.

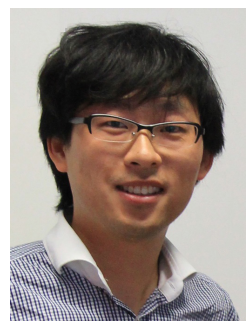

Gaojie Chen (S'09 - M'12) received the B.Eng. and B.Ec. degrees in electrical information engineering and international economics and trade from Northwest University, China, in 2006, and the M.Sc. (Hons.) and Ph.D. degrees in electrical and electronic engineering from Loughborough University, Loughborough, U.K., in 2008 and 2012, respectively. From 2008 to 2009, he was a Software Engineering with DTmobile, Beijing, China, and from 2012 to 2013, he was a Research Associate with the School of Electronic, Electrical and Systems Engineering, Loughborough University. He was a Research Fellow with 5GIC, Faculty of Engineering and Physical Sciences, University of Surrey, U.K., from 2014 to 2015. Then he was a Research Associate with the Department of Engineering Science, University of Oxford, U.K., from 2015 to 2018. He is currently a Lecturer with the Department of Engineering, University of Leicester, U.K. His current research interests include information theory, wireless communications, cooperative communications, cognitive radio, secrecy communication, and random geometric networks.

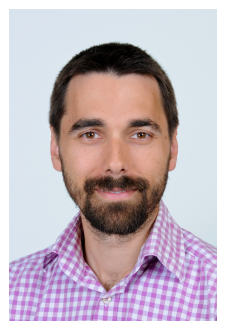

Justin P. Coon (S'02 - M'05 - SM'10) received the B.Sc. degree (Hons.) in electrical engineering from the Calhoun Honours College, Clemson University, USA, and the Ph.D. degree in communications from the University of Bristol, U.K., in 2000 and 2005, respectively. In 2004, he joined as a Research Engineer with the Bristol-based Telecommunications Research Laboratory (TRL), Toshiba Research Europe Ltd., where he was involved in research on a broad range of communication technologies and theories, including single- and multi-carrier modulation techniques, estimation and detection, diversity methods, and system performance analysis and networks. He held the research manager position from 2010 to 2013, during which time he led all theoretical and applied research on the physical layer at TRL. He was a Visiting Fellow with the School of Mathematics, University of Bristol, from 2010 to 2012, where he held a reader position with the Department of Electrical and Electronic Engineering from 2012 to 2013. He joined the University of Oxford in 2013, where he is currently an Associate Professor with the Department of Engineering Science and a Tutorial Fellow of Oriel College.

$\mathrm{He}$ is the Technical Manager of the EU FP7 project DIWINE. He has authored in excess of 100 papers in leading international journals and conferences, and is a named inventor on over 30 patents. His research interests include communication theory, information theory, and network theory. Dr Coon was a recipient of TRLs Distinguished Research Award for his work on block-spread CDMA, aspects of which have been adopted as mandatory features in the 3GPP LTE Rel-8 standard. He was also a co-recipient of two best paper awards at the ISWCS 2013 and the EuCNC 2014. He received the award for Outstanding Contribution in 2014. He has served as an Editor for the IEEE TRANSACTIONS ON WIRELESS COMMUNICATIONS from 2007 to 2013, the IEEE TRANSACTIONS ON VeHICULAR TECHNOLOGY from 2013 to 2016. He has been serving as an Editor for the IEEE WIRELESS COMMUNICATIONS LETTERS since 2016 and the IEEE COMMUNICATIONS LETTERS since 2017. 